\title{
Une « voie ignorée » des études africaines d'Alain Locke à Melville Herskovits et Ralph Bunche
}

"The Road not Taken" of African Studies: From Alain Locke to Melville

Herskovits and Ralph Bunche

\section{Anthony Mangeon}

\section{OpenEdition}

\section{Journals}

Édition électronique

URL : https://journals.openedition.org/etudesafricaines/16292

DOI : 10.4000/etudesafricaines. 16292

ISSN : $1777-5353$

\section{Éditeur}

Éditions de l'EHESS

\section{Édition imprimée}

Date de publication : 20 novembre 2010

Pagination : 619-656

ISBN : 978-2-7132-2252-8

ISSN : 0008-0055

\section{Référence électronique}

Anthony Mangeon, «Une « voie ignorée » des études africaines d'Alain Locke à Melville Herskovits et

Ralph Bunche », Cahiers d'études africaines [En ligne], 198-199-200 | 2010, mis en ligne le 02 janvier 2013, consulté le 23 avril 2022. URL : http://journals.openedition.org/etudesafricaines/16292 ; DOI https://doi.org/10.4000/etudesafricaines.16292

\section{Ce document a été généré automatiquement le 23 avril 2022}

(c) Cahiers d'Études africaines 


\title{
Une « voie ignorée » des études africaines d'Alain Locke à Melville Herskovits et Ralph Bunche
}

\author{
"The Road not Taken" of African Studies: From Alain Locke to Melville
} Herskovits and Ralph Bunche

Anthony Mangeon

Dans l'histoire des African Studies, le philosophe afro-américain Alain Leroy Locke (1885-1954) est comme L'Homme invisible du fameux roman de Ralph Ellison. Son nom n'apparait jamais, et ses contributions aux débats africanistes de son époque sont aujourd'hui largement ignorées. Ses conférences francophones sur «Le rôle du Nègre dans la culture des Amériques ", initialement délivrées en Haïti en 1943, viennent cependant d'être rééditées, et elles révèlent son grand intérêt pour les études africaines. Locke consacre notamment une trentaine de pages à la question de "l'héritage africain et sa signification ", où il cite abondamment l'anthropologue Melville Herskovits (1895-1963). Ses six conférences analysent également les notions de race, de culture et de démocratie, dans les contextes respectivement coloniaux et postcoloniaux de l'Afrique et de l'Amérique, et le philosophe mentionne alors un de ses collègues de l'université d'Howard, le Dr Ralph Bunche (1903-1971), comme «une autorité en matière d'administration coloniale, qui est membre du Bureau des Services Stratégiques et Premier Consultant de sa section africaine » (Locke 2009 : 105).

2 De façon plus générale, un rapide survol de ses publications est riche d'enseignements. Sous sa direction, le numéro spécial du Survey Graphic - «Harlem, Mecca of the New Negro » - avait en mars 1925 lancé le mouvement des « Nègres Nouveaux » en tâchant de valoriser également leurs racines africaines. Une autre livraison de ce même magazine permit à Locke d'inaugurer, en novembre 1942, un questionnement qu'on qualifierait aujourd'hui de postcolonial. Intitulé «Color, Unfinished Business of Democracy ", ce numéro s'articule en effet sur le grand écart entre les principes démocratiques de l'Occident et leurs multiples dévoiements. Locke y expose notamment un double paradoxe: l'Amérique blanche entend défendre, de par le 
monde, un idéal démocratique dont elle dénie la jouissance à ses concitoyens noirs, tandis que la France républicaine voit, à la même époque, son héritage juridique et politique essentiellement sauvegardé, à l'Équateur, par des sujets africains qui n'en bénéficient nullement (Locke 2009 : 180).

Par-delà ces éditions du Survey Graphic, Locke coordonna deux importantes anthologies. The New Negro vint ainsi étoffer, en novembre 1925, le magazine du mois de mars, avec de nouveaux textes littéraires consacrés à l'Afrique et un grand essai d'histoire globale signé Du Bois'. De son côté, le numéro "Color » sera en 1942 suivi de When Peoples Meet, une collection de textes historiques, anthropologiques et sociologiques où, sur plus de sept cents pages, les exemples africains abondent au fil des cinq parties du volume (Locke 1942b). On peut ainsi découvrir une double trame au sein des différentes entreprises éditoriales d'Alain Locke : l'Afrique apparaît tout à la fois comme distante et constitutive de l'identité syncrétique des Afro-américains, et la question raciale se trouve constamment abordée à une double échelle, américaine et africaine ou nationale et internationale.

4 Je propose donc, dans les pages qui suivent, de revisiter d'abord les différents textes qu'Alain Locke consacra à l'Afrique - en particulier les divers memoranda par lesquels il défendit ardemment la création d'un institut d'études africaines à Howard University et d'explorer à leur suite les relations qu'il entretint avec l'anthropologue Melville $\mathrm{J}$. Herskovits et le politologue Ralph J. Bunche, dont les objets de recherche furent, ainsi que nous le verrons, étroitement liés aux propositions africanistes du philosophe. Dans ses constantes et dans ses inflexions, tout comme dans les influences qu'il reçut ou exerça en retour, l'itinéraire d'Alain Locke relève en effet du paradoxe : alors qu'il fut un des principaux acteurs de la "Negro Renaissance ", considérée aujourd'hui comme l'un des grands mouvements culturels de l'Entre-deux-guerres, Locke ne parvint pas à installer durablement les études africaines dans le monde académique noir, et son projet africaniste demeure largement oublié ou méconnu aujourd'hui, de part et d'autre de l'Atlantique. Comment expliquer cette « voie non prise » des African Studies dans la première moitié du $\mathrm{xx}^{\mathrm{e}}$ siècle ? $^{2}$. Quels étaient ses antécédents, ses modèles concurrentiels? Faut-il l'appréhender comme un échec, ou les ambitions africanistes d'Alain Locke et de certains de ses collègues de l'université d'Howard ont-elles connu des prolongements ailleurs et plus tard, au-delà de l'Entre-deux-guerres et du cercle restreint de l'intelligentsia noire américaine?

\section{Genèses d'une vocation africaine}

$5 \quad$ L'intérêt profond qu'eut toute sa vie Alain Locke pour les études africaines trouve ses sources dans son histoire familiale et dans son propre parcours d'" intellectuel noir " au tournant du $\mathrm{xx}^{\mathrm{e}}$ siècle. Enfant unique de deux éducateurs afro-américains, Locke naît en 1885 à Philadelphie, dans une famille qui, de part et d'autre, a participé à la construction du Liberia. Du côté paternel, le grand-père, Ishmael Locke, était un Noir libre qui devint missionnaire à Freetown, où il créa des établissements scolaires et rencontra sa future épouse, Mathilda Saunders, elle-même éducatrice et née au Liberia d'une mère africaine et d'un père allemand. Du côté maternel, la grand-mère de Locke, Sarah Shorter Hawkins - auprès de laquelle il grandira - avait également vécu et enseigné plusieurs années en Afrique (Harris \& Molesworth 2008: 5-7). Dans ce contexte « éclairé » - notamment par les idées d'Edward Wilmot Blyden (1832-1912) et 
d'Alexander Crummell (1819-1898) - il était attendu que Locke visât à l'excellence académique. Ainsi intégra-t-il à la suite la Philadelphia School of Pedagogy (1902-1904), l'Université d'Harvard (1904-1907), puis celles d'Oxford (1907-1910) et de Berlin (1910-1911). C'est en qualité de premier Rhodes Scholar noir américain qu'il vint étudier en Europe. Si l'homme d'affaires et bâtisseur d'empires Cecil J. Rhodes (1853-1902) avait $\mathrm{pu}$ donner son nom à une prestigieuse bourse universitaire, il le devait à sa fortune amassée en Afrique du Sud. Il paraissait donc naturel à Locke que cette dernière bénéficiât à un "fils naturel de l'Afrique " : ainsi justifia-t-il, du moins, sa candidature auprès de la presse américaine alors qu'en raison de sa couleur de peau il rencontrait des difficultés pour être admis dans l'un des collèges d'Oxford (Stewart 1979: 105; Harris 1989 : 294). L'expérience de la discrimination pousse alors le jeune étudiant afroaméricain à se lier d'amitié avec d'autres gens de couleur issus des colonies britanniques. Il rencontre d'abord Lala Har Dayal (1884-1939), un étudiant hindou, et bientôt le Sud-Africain Pixley Isaka Seme (1881-1951) qui, après un séjour à l'Université de Columbia (New York), suivait en Angleterre des cours de droit (Harris \& Molesworth 2008: 145). Har Dayal et Seme devaient par la suite jouer un rôle moteur dans l'émergence de partis anti-colonialistes dans leurs pays d'origine (Boahen 1987: 70 ; Locke 1992 : XXXVII) mais à Oxford ils étaient déjà, avec d'autres, les fers de lance d'une nouvelle génération militante qui alliait désormais cosmopolitisme et nationalisme culturel dans l'idée - revisitée - de « renaissance ». Créé en juin 1909, le magazine The Oxford Cosmopolitan sera leur première tribune (Stewart 1979 : 119-120). La même année, Locke élabore ce que sa mère appelle, dans une lettre de février 1910, son "plan colossal $»^{3}$ : dans la lignée des grands explorateurs, et sur le modèle d'un journalisme engagé qui entend désormais contribuer à l'essor des sciences sociales ${ }^{4}$, le jeune licencié projette un grand voyage de deux ans en Afrique et au Moyen-Orient pour y étudier les différentes attitudes nationales sur la question raciale ${ }^{5}$. Consultable dans ses archives à l'Université d'Howard, le «synopsis d'articles sur les conditions sociales et politiques en Orient et en Afrique » donne effectivement le vertige. Distribué en trois sections, le périple était censé suivre l'itinéraire suivant :

«Constantinople, Smyrna, Aleppo, Bagdad, Damascus (by caravan), Beyrout, Jerusalem, Suez, Cairo, Alexandria, to Assouan, Khartoum, Gondar, to Abyssinia, Adowa, Ras Dashan, to the Coast and either to Aden and the following Eastern Itinerary or an immediate completion of the African section (section 3).

Section 2. Aden to Bombay, Baroda, through the Rajpoctana to Delhi, through the Seik States to Lahore, Cashmere, Simla, Kamat, Nepal, Lucknow, Benares, Patna, Calcutta, through the Deccan to Hyderabad, Madras, Mysore, Ceylon: Kandy, Colombo and the mountain region.

Section 3. Somali Land, Galla Land, Uganda, Congo Free State, German East Africa, Zululand, Natal, Bechunaland, Cape Colony, West Coast Africa $»^{6}$.

6 Les articles prévus devaient se répartir en deux genres, socio-politique et descriptif. Dans la première catégorie, Locke proposait une vingtaine de sujets, qui auraient présenté le Congrès des jeunes Égyptiens, celui des jeunes Turcs ou celui des Nationalistes indiens; il eût abordé les plans sionistes de colonisation de la vallée du Tigre, la recrudescence de l'islam en Inde, celle de l'Église abyssinienne en Afrique de l'Est; les politiques coloniales au Congo, en Afrique de l'Est et en Afrique du Sud auraient été également étudiées, parallèlement aux attitudes missionnaires européennes et américaines envers les divers peuples africains. Les articles descriptifs devaient quant à eux "privilégier la couleur locale sans faire violence à la vie indigène »: Locke imaginait divers récits de "journées " passées dans les rues de 
Constantinople ou dans un mariage égyptien, des visites à la cour de l'empereur d'Abyssinie puis à celles de divers maharajahs indiens, des scènes de chasse ou d'excursions montagnardes... Parmi les journaux intéressés par son périple, il cite le New York Evening Post, le Chicago Tribune, les magazines Colliers et The Outlook. Locke sollicita l'intercession de Booker T. Washington (1856-1915) pour obtenir d'autres soutiens financiers, et ce dernier le recommanda pour une bourse de voyages ( Kahn Traveling Fellowship ») en expliquant comment un tel projet permettrait d'« explorer les relations raciales à l'échelle planétaire ${ }^{7}$. Malheureusement, Locke n'obtint pas ce financement, prioritairement destiné à des voyageurs qui avaient déjà une réputation établie d'enseignants ou de reporters (Harris \& Molesworth 2008 : 119). Son projet d'un africanisme mâtiné d'orientalisme, et tour à tour littéraire et sociologique, fit donc long feu, mais il n'en préfigurait pas moins, dans une certaine mesure, les journaux de bord et les récits par lesquels Michel Leiris (L'Afrique fantôme, 1934) et Georges Balandier (Afrique ambiguë, 1957) allaient imprimer leur marque sur l'ethnologie et la sociologie africanistes en France.

7 Son étude des relations raciales, Locke la conduira dans une série de cinq conférences publiques données à l'Université d'Howard, en 1915 et 1916, et publiées en 1992 sous le titre de Race Contacts and Interracial Relations. Son propos s'y inspire de la psychologie sociale (en particulier de Gabriel Tarde et de Georg Simmel) pour analyser l'idée de race comme la cristallisation d'un " sentiment d'appartenance » (" kinship feeling ») exacerbé par les situations de contact culturel et de concurrence économique. S'il prête attention à cette dimension psychologique, fondant sur elle la possibilité du nationalisme culturel noir américain, le conférencier n'en critique pas moins les usages politiques et idéologiques de la race, dont l'idée sert surtout à justifier les pratiques discriminatoires et inégalitaires inhérentes à toute domination sociale ou coloniale. Faute d'avoir pu voyager en Afrique, comme il le souhaitait ardemment, le jeune philosophe s'était résolu à une carrière universitaire, mais dans l'Amérique ségréguée de son époque, il ne put obtenir de poste ailleurs qu'à "l'université nègre » d'Howard. La condition noire aux États-Unis redevint ainsi son terrain le plus familier - avant que les années 1920 ne viennent relancer ses ambitions africanistes.

\section{«Back to Africa »}

8 L'immédiat après-guerre offre en effet un contexte nouveau: les horreurs des tranchées ont violemment réfuté le positivisme industriel, qui conjuguait ensemble progrès matériel et supériorité morale ; à rebours, une vogue primitiviste et relativiste s'empare de l'Occident. La participation des troupes noires américaines et africaines au conflit européen a par ailleurs généré un regain de fierté, mais aussi, bientôt, d'autres motifs de déception: loin d'être reconnus, les droits et les espoirs d'égalité sont bafoués ; les lynchages se multiplient aux États-Unis tandis que, de part et d'autre de l'Atlantique, les pensions des Anciens combattants noirs sont nettement inférieures à celles de leurs frères d'armes d'origine européenne (Du Bois 1919; Senghor 1927; Dewitte 1985 : 148).

9 Des idéologies nouvelles manifestent un intérêt croissant pour le sort des colonies africaines. Organisé à Paris en février 1919, au moment même où se discute le traité de Versailles, le premier congrès panafricain réclame la création d'un grand État centrafricain, qui rassemblerait les anciennes colonies allemandes, du Cameroun au 
Tanganyika en passant par le Togo, le Rwanda et le Burundi, et qui serait directement administré par la Société des Nations. Ce projet échoue, mais son idée d'un fidéicommis international inspire le système des mandats confiés à la France et à la GrandeBretagne, aux fins d'assurer le développement des populations concernées jusqu'à leur complète autonomie (Locke 1924b : 38 ; Padmore 1960 : 135-136; Lewis 1993 : 576-577). De son côté, le Jamaïcain Marcus Garvey (1887-1940) lance, avec l'Universal Negro Improvement Association, son mouvement d'" un retour en Afrique » ("Back to Africa ») dont il s'auto-proclame président provisoire en 1922. Parallèlement, la Révolution soviétique a marqué, en Russie, le triomphe du marxisme-léninisme, dont les Thèses préliminaires sur la question nationale et coloniale dénoncent, en 1920, l'impérialisme comme le stade suprême du capitalisme dont il s'agit désormais de précipiter la chute. Le doute sur le bien-fondé de la colonisation s'immisce jusque dans les rangs de ses partisans puisqu'en 1921, un administrateur d'origine guyanaise, René Maran, obtient le prix Goncourt pour Batouala, véritable roman nègre, dont la préface constitue un véritable brûlot à l'encontre de l'action coloniale française en Afrique.

Très attentif à ces évolutions, Alain Locke - qui est entre-temps devenu chef du département de philosophie à Howard, et une autorité intellectuelle respectée - tente d'en tirer parti pour promouvoir les études africaines. Il multiplie d'abord les recensions et les articles dans la presse et dans des revues engagées comme Opportunity ou The Survey ${ }^{8}$. De 1923 à avril 1927, il alterne ainsi des comptes rendus sur l'art nègre avec des recensions de la littérature coloniale française ou britannique et des réflexions d'ordre général sur les questions raciales et coloniales (Locke 1923a, 1924b, d, e, f, 1925a, c, d, e). Il rédige et publie également deux importants reportages de terrain sur l'occupation de la Ruhr par les troupes noires françaises en 1923 et sur son voyage, la même année, au Soudan et en Égypte (ibid. : 1924a, c).

Dans ces différents textes, Locke prend acte du regain d'intérêt en faveur de l'Afrique pour souligner divers décalages entre l'Europe et l'Amérique. De plus en plus reconnu sur le vieux continent, l'art nègre reste en effet largement ignoré du Nouveau Monde à l'exception de quelques collectionneurs privés. Constater ses influences sur la création contemporaine lui permet alors de redéfinir la notion d'« art primitif ", d'une conception strictement évolutionniste à une approche plus historique :

«Equally important with this newer aesthetic appreciation is the newer archeological revaluation. Negro art is no longer taken as the expression of a uniformly primitive and prematurely arrested stage of culture. It is now seen as having passed through many diverse phases, as having undergone several classical developments, and as illustrating several divergent types of art evolution. The theory of evolution has put art into a scientific straight-jacket, and African art has had to fit in with its rigid preconceptions. [...] Perhaps the most important effect of interpretations like these is to break down the invidious distinction between art with a capital for European forms of expression and "exotic" and "primitive" art for the art expressions of other peoples. Technically speaking an art is primitive in any phase before it has mastered its idiom of expression, and classic when it has arrived at maturity and before it has begun to decline. Similarly art is exotic with relation only to its relative incommensurability with other cultures, in influencing them at all vitally it ceases to be exotic. [...] Art is universally organic ».(Locke 1924e : 136 ; Stewart $1983: 133)$

Dans cette nouvelle perspective, l'art africain ferait office d'âge classique, dont les artistes afro-américains devraient s'inspirer pour promouvoir une nouvelle «Renaissance» (Locke 1927a: 60; Stewart 1983: 139). La visée est cependant 
identitaire, qui cherche à compenser les effets dévastateurs du déracinement et de la dévalorisation sociale en s'ancrant dans un prestigieux capital symbolique et culturel :

« Nothing is more galvanizing than the sense of a cultural past. This at least the intelligent presentation of African art will supply to us. Without other more direct influence even, a great cultural impetus would thus be given ».(Locke 1924e : 138; Stewart $1983: 135)$

Telle que promue par Locke, l'idée d'une "Renaissance nègre " sera étroitement associée à cette idée de "capitalisation ". L'anthologie The New Negro, An Interpretation (1925) en constitue une première incarnation, avec ses poèmes, ses articles, ses reproductions d'art et ses bibliographies consacrées à l'Afrique. En dépit de quelques concessions au primitivisme ambiant, les dessins d'Aaron Douglas (1898-1979) illustrent comment les modes de représentation en Afrique (de l'art égyptien aux motifs géométriques abstraits, en passant par la stylisation des masques et des sculptures) influencent la créativité afro-américaine. L'Égypte ancienne occupe de fait une place particulière dans la perspective revivaliste de Locke : alors qu'il voyage dans le nord-est de l'Afrique, à l'automne 1923, en compagnie de son ami sud-africain Pixley Isaka Seme, l'universitaire afro-américain a l'opportunité d'être parmi les premières personnes à visiter la tombe de Tout-Ankh-Amon au moment de sa réouverture, un an après sa découverte. Loin de mettre les accomplissements des pharaons d'Égypte au seul crédit de leur négritude, le chroniqueur d'« Impressions of Luxor » (Locke 1924c) y voit l'une des plus « extraordinaires manifestations d'un art composite » qui aurait atteint « une expression presque classique, par l'équilibre de ses divers éléments et par sa perfection ", et qui incarnerait ainsi « la première grande renaissance culturelle »:

«What social conditions, what cultural influences produced it? [...] Wherever the impetus came from, it was, we must remember, focalized here in an African setting and in a polyglot civilization that must have included more African, and possibly even Negro components than will ordinarily be admitted, so wide-spread is the impression that nothing profoundly cultural can come out of Africa even though it may be found in Africa. [...] What seems to me most likely as an outcome after all the hypotheses have had their chance will be a confirmation of the broadest and most natural of all hypotheses, - namely, that great cultures are the result invariably of the fusion of several cultures, [...] the fermenting of one civilization by another. Once we recognize this principle of the cross-fertilization of cultures, [...] our science of man will have been clarified of its greatest pollution, ethnic bias in terms of cultural prejudice ».(Locke 1924c : 77-78).

En empruntant à l'anthropologie culturelle de Franz Boas (1911) l'idée d'un syncrétisme originaire de toutes les cultures, Locke plaide pour une nouvelle approche des civilisations africaines, où le réalisme et l'étude des contacts de civilisation primeraient désormais sur l'exotisme et le primitivisme. C'est ainsi qu'il en vient à célébrer la "nouvelle littérature coloniale de la France» qui, sous la plume de René Maran (1887-1960) et de Lucie Cousturier (1870-1925), manifeste une nouvelle exigence d'objectivité et de connaissance de l'Afrique. S'il rend aussi des hommages appuyés au "garveyisme » et au panafricanisme, pour " avoir agité, au plus profond de l'esprit de la race, l'idée d'une coopération à grande échelle entre les branches différentes et séparées des peuples nègres " (1924b : 38), Locke déplore cependant que ces élans de solidarité procèdent d'un rapport à l'Afrique strictement affectif, où le mélange de romantisme sentimental et de condescendance missionnaire ne diffère guère, en définitive, de l'idéalisme humanitaire et du sentiment de supériorité qui justifièrent en Europe la colonisation de l'Afrique. 


\begin{abstract}
« Except from the point of view of religious missionarism, it has been until recently almost impossible to cultivate generally in the mind of the American Negro an abiding and serious interest in Africa. [...] We need to be the first of all Westerners to rid ourselves of the insulting prejudice, the insufferable bias of the attitude of "civilizing Africa", - for she is not only our mother but in the light of most recent science is beginning to appear as the mother of civilization in general. [...] Our interests are fed on sentiment, and not with knowledge. Our first duty is to cultivate every opportunity for the diffusion among us of the knowledge of Africa both of today and of the past. Travel, exchange of students, the spread of journalistic and academic information are for the moment of paramount importance. In a decade in which the study of African art and archeology has come to the very forefront of scholarship, it is both a reproach and an handicap to have no recognized experts of our own in these fields. Instead of being reluctant, our Negro colleges should be eager to develop special scholarship in theses directions [...]. The pioneer work of the Journal of Negro History, under Dr Carter G. Woodson, and of Howard University in the courses of the history of African civilizations, under Mr Leo Hansberry, deserve not mere passing interest and praise but the financial support of the people and the active participation of the talented tenth ". (Locke 1924b : 37-39)
\end{abstract}

Une telle insistance est évidemment motivée. En se rendant en Égypte, Locke avait pu nouer des liens privilégiés avec l'Institut français d'archéologie orientale, dont le directeur Georges Foucart (1865-1943) s'était engagé à développer certains programmes de recherche en partenariat avec Howard University (Locke 1924c: 76). Locke rencontre également, à la même époque, Maurice Delafosse (1870-1926) qui le fait élire correspondant étranger de l'Académie des sciences coloniales (créée en 1922) et qui échange avec lui plusieurs lettres ${ }^{10}$. Initialement pourvu d'une formation d'orientaliste - il traduira notamment avec son beau-père, Octave Houdas (1840-1916), le Tarikh EsSoudan en 1913 - Delafosse avait rédigé plusieurs manuels linguistiques et d'importants travaux ethnographiques et historiques lors de sa carrière d'administrateur colonial en Afrique subsaharienne (Amselle \& Sibeud 1998). Il était à cet égard une autorité appréciée par l'Amérique noire : le grand historien William Du Bois (1868-1963) s'était inspiré des écrits de l'africaniste français pour rédiger The Negro (1915), un livre de vulgarisation dont la lecture suscita une véritable vocation africaniste chez Leo Hansberry (1894-1965), un étudiant noir de Harvard, dont les premiers écrits allaient à leur tour reposer sur Delafosse et Leo Frobenius (1873-1938) (Hansberry 1921 ; Harris 1981 : 7). Locke fait lui-même souvent référence à Delafosse dans ses articles des années 1920 , et par la suite il ne manquera pas de signaler la traduction posthume, en anglais, de certains de ses livres (Locke 1924d : 109, 1925f : 447, $1932:$ 41, $2009: 26,30,145$ ).

Les premières études africaines de l'Amérique noire s'inscrivent donc ouvertement dans la tradition naissante de l'africanisme français, mais elles poursuivent également un autre agenda que la simple exhumation d'un prestigieux passé africain à des fins d'édification. En s'intéressant aux politiques coloniales européennes, et à leurs traitements respectifs de la différence raciale, elles visent à fournir un cadre comparatif, et donc à soulever la possibilité d'alternatives à la ségrégation, par-delà les contrastes saillants entre l'idéologie assimilationniste de la France et les pratiques discriminatoires de la Grande-Bretagne ou des États-Unis. Là réside tout le sens de l'article que Locke consacre, en 1924, à l'occupation de la Rhénanie par les troupes coloniales noires, avec la bénédiction de Delafosse ${ }^{11}$. Et lorsque l'écrivain et publiciste René Maran dénonce, en première page de son journal Les Continents, la réalité du racisme métropolitain ou les aberrations du régime de l'indigénat dans les colonies, 
accusant Locke d'avoir été « victime d'un mirage » quant à la libéralité française sur les questions raciales et coloniales, ce dernier répond en termes explicitement tactiques à cette « lettre ouverte» :

«Our battle is on several fronts, my dear M. Maran. We have perforce different immediate objectives, there are some nasty salients yet to be straightened out that at times bring us almost back to back when we should be standing shoulder to shoulder, [...] but as day by day the alignment of our problem becomes more and more world-encircling, we will learn to consolidate our positions and heed one general command. [...] As for my article, my firing-range was set from our own trenches and for a very special purpose. I was not discussing French policy in Africa, but merely the French treatment of her Negro soldiers in Europe. At the time I was primarily concerned with contrasting this treatment of the man of color in the armies of France with that of our own American army [...]. If you knew our position in America by contrast of actual experience, you would understand why we praise in certain ways, as we, when you know your position, can quite comprehend why you must censure. Some things are possible for the black man in France which are not yet possible for him in America ; some advantages and gains that we have in America have not as yet been achieved under France. [...] Which but means that we fight one another's battle even in fighting our own ».(Locke 1924f : 262-263)

\section{Les avatars d'un projet africaniste}

Loin d'endosser la politique coloniale française, Locke projette en effet d'en exposer les contradictions au sein d'une vaste enquête de terrain. En mai 1927, il soumet à la Foreign Policy Association - une excroissance des ligues anti-impérialistes fondée en 1918, et toujours active de nos jours - un mémorandum où il motive en ces termes son désir d'étudier le nouveau système des mandats africains mis en place par la Société des Nations, conformément à l'article 22 du traité de Versailles :

«The administration of mandates in the spirit of international guardianship of the right of undeveloped peoples and their preparatory tutelage for participation in government and constructive self-adjustment is one of the most important and progressive aspects of the work of the League of Nations. Public opinion in the United States on this phase of world politics is uninformed and even disinterested; and this is especially true of public opinion among Negroes, who, however, should have the keenest interest in this phase of international policy both because of their relationship to the African peoples under mandate control and because of the similarity of some of the problems to those of race relations in the United States. [...] The two Committees of the League whose deliberations and findings would come under special observation would naturally be the Mandates Commission and the newly formed Advisory Committee on Native Labor. [...] In such a study, the program of native education, broadly interpreted to include official educational agencies and programs in health, sanitation, land adjustment and cultivation, as well as the governmental attitude toward tribal life and institutions, and toward cooperation with established missionary and other educational agencies, ought in my judgement be made the pivot of the inquiry rather than the mere political administration of the mandates. [...] If authorized by the FPA to make the Geneva study, I would also be willing to pursue such field study later in the mandated territories and elsewhere in Africa ».(« Memorandum ; Foreign Policy Association; Alain Locke re : African Mandates Study Project $»: 1)$

Ce projet, qui rappelle le "schème colossal » proposé par Locke à la Fondation Kahn dans les années 1910, est à nouveau soumis à une organisation non gouvernementale car depuis 1925, Locke n'enseigne plus à l'Université d'Howard. Il en fut en effet licencié pour avoir tout ensemble défendu la création d'un Institut d'études africaines 
et revendiqué l'égalité salariale entre professeurs noirs et professeurs blancs ${ }^{12}$. Ce congé forcé lui offrira, de fait, l'opportunité d'éditer deux nouvelles anthologies de littérature en 1927 (Four Negro Poets ; Plays of Negro Life), et d'engager parallèlement une étude des systèmes coloniaux africains et de leur évolution. Mais en dépit d'abondantes notes de lecture sur le travail forcé en Afrique $^{13}$, et malgré un rapport de trente-six pages sur « le système des mandats, nouveau code de l'empire $»^{14}$, le chercheur se verra finalement refuser toute possibilité de financement pour se rendre, ainsi qu'il le projetait, au Dahomey et au Togo puis en Afrique du Sud afin d'y approfondir son travail sur le terrain ${ }^{15}$.

Locke n'est pas le seul Afro-américain à s'intéresser, à l'époque, au système des mandats: Rayford Logan (1897-1982), un historien diplômé de Williams College qui avait combattu en France et participé aux divers congrès panafricains des années $1920^{16}$, publie en effet, en octobre 1928, un article de cinquante pages dans The Journal of Negro History sur «Le fonctionnement du système des mandats en Afrique " (Logan 1928). S'étonnant, avec une froide ironie, que le président américain Wilson se soucie « du bien-être des Bantous, des Ouolofs, des Mandingues, des Doualas et d'autres tribus dont il n'a jamais entendu parler, alors qu'il demeure sourd aux plaintes des paysans noirs dans son propre pays " (ibid. : 427), Logan a tôt fait de montrer que derrière les principes proclamés du bonheur et du droit des peuples à l'autonomie, au libre échange et à la libre circulation, ce sont en réalité les méthodes impérialistes du monopole et de l'exploitation économiques qui se trouvent reconduites par les pays mandataires, faute d'un véritable système international de contrôle et de sanctions (ibid. : 438, 442). Cet article retiendra l'attention de Paul Kellogg, directeur du Survey et fondateur de la Foreign Policy Association (FPA), en même temps qu'il suscitera l'admiration de Locke : sa publication pourrait donc à elle seule expliquer pourquoi la FPA ne jugeait plus nécessaire de publier le rapport du philosophe. En novembre 1928, Locke correspond avec Kellogg et Logan dans l'espoir de pouvoir faire recruter ce dernier à Howard University et de le faire participer à la création d'un centre d'études africaines (Janken 1993 : 79). Mais le projet échoue et Logan n'intégrera finalement Howard qu'en 1938. De son côté Locke avait été réembauché par Mordecai Johnson (1890-1976), le nouveau président (noir) de l'université, durant l'automne 1927. Il avait alors soumis au conseil d'administration un nouveau "Memorandum pour un Département d'études africaines ». Daté du 5 juin 1928, ce projet ne sera pas plus agréé qu'en 1924, mais il mérite d'être relu car son rejet soulève plusieurs questions : quelles fins, quelles formes et quelles orientations Locke donnait-il aux études africaines pour rencontrer de telles résistances? Son projet africaniste était-il mal conçu ou bien s'avérait-il, au contraire, trop en avance sur son temps? Enfin, n'a-t-il vraiment laissé aucune empreinte sur le devenir des études africaines aux États-Unis?

Dès l'introduction, Locke souligne la nécessité d'« étudier les problèmes de la vie nègre » et, partant, la question raciale à une échelle globale et non plus nationale. $\mathrm{Ce}$ thème constant de sa réflexion constitue de fait son argument majeur en faveur des études africaines. La structuration du département censé les abriter est par ailleurs tout à fait significative : en mutualisant les enseignements dispensés dans un certain nombre de disciplines universitaires alors représentées à Howard University (telles que l'histoire, la sociologie, la psychologie, l'anthropologie, les sciences politiques, la philosophie), l'institut prendrait pour modèle l'International Institute of African Languages and Cultures (IIALC), alors tout récemment fondé à Londres (1926), mais il 
tâcherait d'éviter toute duplication avec ce dernier en se concentrant sur «six branches de recherche et d'étude » :

«1. The field of African and Negro History : to be developed by a man of research training and calibre who would be directly connected with the department of History [...].

2. The field of Anthropology (especially what is known as Cultural Anthropology): to develop the special study of African origins and culture, to be developed under either the department of History or the department of Sociology or separately.

3. The field of Ethnology: with special reference to the investigation of race types and the problem of race traits and with more specific reference to folklore, African and native American.

4. The field of Colonial History and Colonial Administration, especially as it applies to Africa : which should be the special academic interest of at least one man in the department of History and Government [...].

5. The field of Education: with special reference to educational programs for indigenous peoples and the building up of comparative data on the educational progress of the Negro in America and elsewhere [...].

6. The field of African Art and Culture : as an interpretation of the contributions of Negro peoples to culture and civilization; - to be best developed perhaps in connection with the work of the department of philosophy ».(Locke 1928a : 2-3)

Dans un autre memorandum, soumis au milieu des années 1930, Locke insiste à nouveau sur la nécessité de développer un curriculum et des recherches dans quatre directions principales: "l'anthropologie physique et culturelle, l'archéologie et l'histoire de l'Afrique antique et médiévale, l'étude des contacts et des conflits culturels à l'échelle inter-ethnique ou au niveau des contacts missionnaires et coloniaux, et pour finir les langues et les littératures africaines $»^{17}$. Outre un certain nombre d'autorités africanistes susceptibles de rejoindre le comité scientifique - parmi lesquelles on compte Charles Seligman (1873-1940), Diedrich Westermann (1875-1956), Henri Labouret (1878-1958) et Bronislaw Malinowski (1884-1942) -, Locke fournit une liste de noms et de cours pouvant d'ores et déjà être intégrés à ce nouveau curriculum. Parmi eux, nous trouvons les enseignements de l'anthropologue allemand Julius Ernst Lips (1895-1950) qui, après avoir émigré aux États-Unis en 1934, avait été recruté par Howard University en $1937^{18}$; viennent ensuite les cours d'histoire antique de Leo Hansberry ${ }^{19}$, et le cours de Ralph Bunche sur la politique coloniale. L'anthropologue afro-américain Allison Davis (1902-1983) est également mentionné20.

Si le modèle de l'Institut international des langues et des civilisations africaines est prégnant dans les préoccupations éducationnelles affichées dès 1928, ou à travers les noms cités pour le comité scientifique, on peut noter trois différences majeures qui révèlent d'emblée certaines faiblesses du projet lockien. Alors que l'Institut de Londres repose sur un puissant réseau académique et politique, rassemblant « onze universités, dix sociétés savantes, deux musées, huit sociétés missionnaires et deux sociétés coloniales » (de L'Estoile 1997 : 23), la structure imaginée par Locke se limite au seul campus d'Howard University, et un flou demeure quant à son appellation, qui hésite entre deux logiques : la logique strictement académique qu'incarne le Département, et la logique plus institutionnelle et politique que représente l'Institut. De surcroît, Locke n'apporte aucune précision sur les possibles ressources financières des études africaines à Howard, alors même que l'IIALc est soutenu par la Fondation américaine Rockefeller, avec « le financement d'un vaste programme de recherche orienté vers les problèmes du "contact des cultures" et du "changement social" en Afrique, qui prévoit notamment l'envoi sur le terrain de jeunes chercheurs» (ibid.: 25 ; Stocking 1985). 
Enfin, l'IIALc se dote d'emblée d'une revue, Africa, et il entend "mobiliser les résultats d'une connaissance scientifique au service de la résolution des problèmes coloniaux, "importants" pour la "vie réelle des peuples africains", ou plus exactement qui sont considérés comme tels par ceux qui "travaillent pour le bien de l'Afrique", c'est-à-dire essentiellement les agents des diverses bureaucraties coloniales, administratives, éducatives ou missionnaires » (de L'Estoile 1997 : 22). Mais dans le contexte américain, les études africaines ne sauraient bénéficier de telles bases ni se justifier d'une telle fin pratique, et les mobiles strictement scientifiques ou symboliques pèsent évidemment moins lourds dès lors qu'il s'agit de mobiliser des capitaux pour les soutenir.

À cette même époque, Locke justifie donc la nécessité des études africaines en les intégrant à une rupture épistémologique à l'œuvre dans les sciences humaines. Dans deux essais respectivement intitulés "The Handicaps of African Study " et "The Transformation of African Studies", il défend en effet l'abandon définitif de l'évolutionnisme en faveur de nouvelles approches, soulignant que « toutes les cultures doivent être analysées de façon plus historique et objective, et selon leurs valeurs propres ou leurs formes intrinsèques ${ }^{21}$. Son propos fait allusion au fonctionnalisme de l'anthropologie sociale britannique, incarné par Malinowski, auquel s'adjoint - via plusieurs citations d'Alexander Goldenweiser (1880-1940) ou de Melville Herskovits la tradition américaine de l'anthropologie culturelle américaine initiée par Franz Boas $^{22}$.

Locke n'est évidemment pas le seul, à l'époque, à tenter d'opérer cette synthèse entre Boas et Malinowski au sein des études africaines. Sur cette question des méthodes comme sur d'importantes directions de recherche, il est en phase avec Herskovits, rencontré au milieu des années 1920 alors que ce dernier, étudiant de Boas, était venu effectuer des mesures craniologiques à l'Université d'Howard pour démontrer l'instabilité des types humains (Gershenhorn 2004: 32-35; Herskovits 1925a, 1925b, 1926; Jackson 1986: 110). S'il s'intéressait au métissage biologique, Herskovits n'accordait guère d'attention, à l'époque, à la question des échanges ou des syncrétismes culturels, considérant plutôt que la communauté noire des États-Unis, loin d'avoir des traits culturels spécifiques, ressemblait à s'y méprendre à l'Amérique blanche, dont elle suivait le modèle malgré sa différence de peau. "The same pattern, only a different shade ! » servait ainsi de leitmotiv à sa contribution au New Negro, dont le titre - «The Negro's Americanism» - exprimait d'emblée sa conviction assimilationniste. Sur l'insistance de Locke, l'anthropologue sera progressivement conduit à s'interroger sur les rémanences africaines dans les cultures noires du Nouveau Monde, au cours de divers terrains qu'il mènera au Surinam, au Dahomey et en Haiiti ${ }^{23}$. Il écrira ensuite The Myth of the Negro Past (Herskovits 1941), et cette magistrale synthèse sur les héritages culturels africains et leurs réinterprétations, transformations et influences dans les sociétés américaines sera de fait saluée comme un événement par de nombreux intellectuels noirs américains (Jackson 1986 : 122). En dépit de ses réserves initiales (Locke 1942c: 87), Locke cite l'ouvrage dans ses conférences haïtiennes de 1943; il y reconnaît en effet le projet africaniste du New Negro dont la conception des études africaines se trouve désormais pleinement assumée par Herskovits.

« To give the Negro an appreciation of his past is to endow him with the confidence in his own position in this country and in the world which he must have, and which he can best attain when he has available a foundation of scientific fact concerning the ancestral cultures of Africa and the survivals of Africanisms in the New World. 
And it must again be emphasized that when such a body of fact, solidly grounded, is established, a ferment must follow which, when this information is diffused over the population as a whole, will influence opinion in general concerning Negro abilities and potentialities, and thus contribute to a lessening of interracial tensions ».(Herskovits $1958: 32$ ) américaine, le monde universitaire est marqué par la ségrégation, et sans pouvoir entrer en concurrence avec les divers établissements de l'enseignement supérieur américain, les universités noires créées au sortir de la Guerre civile (Howard, Fisk, Atlanta, etc.) restent fortement dépendantes de financements extérieurs, qu'ils soient publics - c'est-à-dire émanant du Congrès - ou privés - c'est-à-dire en provenance de fondations philanthropiques comme le Julius Rosenwald Fund, le Phelps-Stockes Fund, la Carnegie Corporation ou le General Education Board de la Rockefeller Foundation. Or toutes ces institutions sont réfractaires aux programmes de recherches qui remettent trop ouvertement en cause l'idéologie culturelle dominante ou le statu quo ségrégationniste (Smith 1994 : 95 ; Gershenhorn 2004 : 131-132). Dans une Amérique qui se veut, à l'époque, l'héritière des White Anglo-Saxon Protestants, promouvoir l'idée de possibles influences africaines sur les us et coutumes d'une partie - même dévalorisée - de la population pose évidemment problème. Dans leurs efforts intégrationnistes, beaucoup d'intellectuels noirs américains - tels le président d'Howard, Mordecai Johnson, ou encore le sociologue Ezra Franklin Frazier (1894-1962) - se méfient également de recherches africanistes qui risquent, selon eux, d'être malignement réinterprétées pour justifier et renforcer, au nom d'irréductibles atavismes raciaux, les politiques actives de la ségrégation et de la discrimination raciales. Une forme d'autocensure règne ainsi dans les universités noires, où l'on évite prudemment de soutenir les projets africanistes d'un Woodson, d'un Hansberry ou d'un Locke (Goggin 1993 : 45 ; Harris 1981 : 5-18 ; Harris \& Molesworth 2008 : 120).

Mais un autre paramètre entre également en ligne de compte: quoiqu'elles s'en défendent, les fondations privées ont en effet tendance à favoriser des chercheurs blancs, qu'elles jugent moins directement concernés ou impliqués, et donc plus distants et objectifs sur toutes ces questions. Herskovits fera lui-même plus tard un argument de ce présupposé en défendant "l'intégration des chercheurs américains » dans les études africaines au nom de leur «distanciation scientifique vis-à-vis des objets africains » (Piriou \& Sibeud 1997 : 14). Les diverses rebuffades endurées par Woodson, Du Bois, Hansberry et Locke mériteraient à elles seules un article, tant leurs divers projets d'Institut africain ou d'Encyclopédie du monde nègre pâtiront des atermoiements du Rosenwald Fund, du Phelps-Stocke Fund ou de la Carnegie Corporation $^{24}$. En dépit de ses difficultés initiales à se faire financer, Herskovits se trouve en revanche assez vite adoubé par les fondations, et il joue alors un rôle ambigu de gardien et de censeur en rapportant de façon systématiquement négative sur divers projets soumis par des intellectuels noirs - jusqu'à ce que le directeur de la Carnegie 
Corporation, Frederick Keppel (1875-1943), lui préfère à son tour le Suédois Gunnar Myrdal (1898-1987) pour conduire, à la fin des années 1930, la grande enquête sur la situation des Noirs aux États-Unis (Myrdal 1944) ${ }^{25}$. Très critique, dans ses déclarations, à l'égard des finalités pratiques ou des orientations politiques conférées à la recherche par les fondations privées ou par les intellectuels noirs, Herskovits assumait dans les faits une position plus ambivalente (Jackson 1986 : 116-117). Sous couvert d'objectivité scientifique et de neutralité en matière d'activisme social, il n'en participe pas moins, en effet, à l'étude financée par la Carnegie Corporation, dont l'intérêt pour la question des survivances africaines est surtout motivé par la volonté de pallier les possibles problèmes de l'assimilation des Noirs dans le creuset américain ${ }^{26}$. Et après avoir contribué à en exclure les principaux ténors du "Talented Tenth » noir américain ${ }^{27}$, Herskovits dominera le champ africaniste américain en étant le premier à publier un livre (The Myth of the Negro Past) dans la série des monographies issues de l'enquête Myrdal. Son insistance sur les survivances et ses efforts ultérieurs pour institutionnaliser les études africaines aux États-Unis peuvent ainsi s'apparenter à une captation du projet africaniste défendu par Alain Locke et par les New Negroes. Mais dans son autre versant - à savoir l'intérêt pour les questions coloniales en Afrique - ce projet connaît aussi certains prolongements dans l'étonnante carrière de Ralph Bunche.

\section{Ralph Bunche, continuateur d'Alain Locke}

28 J'ai déjà souligné quelles perspectives sociopolitiques visait Locke en défendant la nécessité d'étudier la question raciale à une échelle globale, et comment son choix d'analyser les diverses politiques d'administration coloniale participait d'une volonté plus large de mettre au jour les divers ressorts de la domination impériale des Blancs sur les Noirs ${ }^{28}$. À la fin des années 1930, Locke établit même d'étroits parallélismes entre la situation des Noirs Américains dans le sud des États-Unis et celle des Africains sous les divers régimes coloniaux :

"In the so-called Black Belt, which is about 60 percent of the Southern area, the Negro population is only a numerical minority by a small margin, and in certain specific areas is really in majority in numbers, though not in economic resources or political power. The one thing to be agreed upon by all schools of thought on the subject is, therefore, that a solution within the status quo is out of the question. For that status quo rests upon political disenfranchisement, economic exploitation, arbitrarily and legally defined bi-racial life in separate schools, public conveyances, churches and other institutional organizations, and a social stigma of "inferiority" to bolster the social policy of "white supremacy". To observers not familiar with the situation in detail, it might be graphically described as an internal colonial status, an ingrowing imperialism based on color caste ».(Locke $2005: 43)$

Locke n'est encore une fois pas le seul à soutenir de telles vues en 1938 : à la même époque, son collègue Ralph Bunche délivre aux universités d'Howard et de Northwestern une conférence sur les relations raciales en Afrique du Sud et aux ÉtatsUnis, où il expose certaines similarités politiques, sociales, et économiques entre les deux pays : ici et là, les Noirs sont traités comme des enfants primitifs, tout juste bons à accomplir les tâches domestiques, agricoles ou ouvrières les moins qualifiées ; s'il existe des divisions au sein de la communauté blanche, entre d'un côté les "Yankees " et les gens du Sud (Southerners), et de l'autre les Anglais et les Boers, la discrimination raciale crée également toute une série d'oppositions entre les Noirs, selon leur degré de 
métissage ; enfin, la ségrégation suscite de part et d'autre des stratégies séparatistes, avec la tentation de s'en tenir à un strict biracialisme sur le plan des affaires ou de l'éducation, ou l'adhésion aux thèses racistes les plus extrémistes sur le plan idéologique (Edgar 1992: 314-315). Ralph Bunche me semble ainsi d'autant mieux abonder dans le sens des propos d'Alain Locke qu'il s'est inscrit dans le prolongement direct de ses divers projets de recherche, ainsi que je voudrais à présent le montrer ${ }^{29}$.

Les deux hommes s'étaient liés d'amitié en 1928, à l'époque où Ralph Bunche, alors brillant diplômé noir de l'Université de Californie et d'Harvard, venait d'être recruté à Howard pour y installer un département de sciences politiques (Urquhart 1993 : 44). Ils avaient ainsi formé, avec d'autres collègues comme l'économiste Abram Harris (1899-1963), le sociologue Ezra Franklin Frazier, et le poète et critique littéraire Sterling Brown (1901-1989), un cercle radical dont les discussions portaient sur les développements des sciences sociales et sur les divers moyens, académiques et militants, de substituer des analyses historiques et économiques aux discours prédominants indexés sur la notion de race (Henry $1990: 53$; Holloway $2002: 17$ ).

Mais, désireux de reprendre ses études doctorales, Bunche candidate en 1931 à une bourse du fond Rosenwald. Son projet initial - une comparaison des politiques raciales aux États-Unis et au Brésil - est rejeté car le directeur de la fondation, Edwin E. Embree (1883-1950), « craint que les Nègres américains ne ramènent des idées dangereuses du Brésil » (Edgar 1992: 5 ; Urquhart 1993 : 51). Bunche présente alors un nouveau projet qui semble directement issu du travail engagé par Locke, quelques années plus tôt, auprès de la Foreign Policy Association : il s'agit en effet d'étudier les mises en acte du système des mandats en comparant les politiques coloniales de la France au Dahomey et au Togo. Grâce aux 2000 dollars finalement accordés par le Rosenwald Fund, Bunche vient à l'automne 1932 passer quatre mois en France et à Genève, avant de se rendre en Afrique de l'Ouest, du 5 novembre 1932 au 15 janvier 1933. Il soutient ensuite, en février 1934, une thèse de philosophie à l'Université d'Harvard, qui lui vaut le titre de premier docteur noir en sciences politiques ainsi qu'un prix d'excellence (Toppan Prize) pour le meilleur travail de l'année en "sciences du gouvernement». Sobrement intitulée French Administration in Togoland and Dahomey, cette recherche s'avoue d'emblée " mue par un profond intérêt pour le développement des peuples assujettis et pour les espoirs que l'avenir leur réserve» (Bunche 1934: 1). Elle s'ancre ainsi dans une filiation panafricaine, mais surtout elle permet d'établir qu'il n'est, dans les faits, aucune différence entre les colonies traditionnelles et les mandats gérés par la France pour le compte de la Société des Nations: leurs populations sont soumises aux mêmes politiques fiscale, éducative et judiciaire, avec un semblable impôt de capitation, une scolarité réduite pour l'essentiel à l'enseignement primaire ou technique et professionnel, et l'exercice de la justice dans les seules mains des administrateurs coloniaux. Bunche dénonce alors ce qu'il appelle "le paradoxe français", où l'on prétend mettre en œuvre une politique assimilationniste tout en restreignant, dans les faits, l'accès à la culture et à la citoyenneté françaises (ibid. : 364). Seuls 97 Dahoméens sont en effet admis au rang de citoyens entre 1912 et 1932, avec de surcroît une différence de statut pour leurs enfants, qui demeurent sujets ou deviennent citoyens selon qu'ils sont nés avant ou après la naturalisation de leurs parents. Pourtant, même ainsi limitée, cette possibilité d'accès à la citoyenneté reste fortement convoitée par les Africains " éduqués ", qui rejettent en masse la culture coloniale limitée dans laquelle on veut les confiner (ibid.: 318). Dans ces revendications des principes et des droits 
promus par les colonisateurs, mais réservés à eux-mêmes et à quelques privilégiés, le politologue identifie précisément les ressorts des futures luttes pour la décolonisation:

«Sooner or later, as the educated class of natives increases in numbers, the French will be confronted with the difficult problem of the colonial administration of backward peoples, viz., that there is no apparent peaceful means of transition to full self-control. This condition is already looming larger on the horizon in neighboring British Gold Coast. The French will be able to postpone this day of reckoning longer than will the British perhaps, because France finds it possible to mollify the élite by giving them racial and social equality. [...] Tribal authority has been broken down deliberately by the French. This may prove to be a blessing in disguise to the native however, for it will make it possible for him to ultimately present a united front in his demands for an increasing share in the control of his own country ».(Bunche 1934 : 422 ; cité également dans Urquhart 1993: 55 et dans Kilson 1990 : 91)

Avec ces divers constats, Bunche se démarque très nettement des perspectives prévalentes à son époque. Il critique tout d'abord le projet éducationnel de Booker T. Washington dont l'insistance sur une formation strictement pratique et technique, à destination des Noirs du sud des États-Unis, sert désormais à justifier les politiques éducatives indigènes en Afrique (Bunche 1934 : 327). Bunche prend aussi ses distances avec Thomas Jesse Jones (1873-1950), influent directeur du Phelps-Stockes Fund et nouveau champion des conceptions utilitaristes dans l'éducation des Noirs. Sa critique des connivences entre colonialisme européen et philanthropie américaine fait écho à celle de Melville Herskovits, qui stigmatise également, à la même époque, la préséance des administrateurs coloniaux sur les chercheurs en sciences sociales dans les études financées par les fondations (Bunche 1936b ; Jackson 1986 : 116).

En déniant toute spécificité négro-américaine ou africaine, sur le plan éducationnel comme sur le plan culturel, le politologue s'avère ensuite un critique acerbe de l'ethnologie africaniste. Dans sa quête de l'africanité, cette dernière fait en effet souvent abstraction de la situation coloniale :

"The colonial anthropologist revels in the discovery of an isolated, untouched primitive culture, which may be analyzed in its condition of pristine purity, and it is not many years since Africa afforded him an excellent playground ».(Bunche 1937 : $639)^{30}$

À rebours, Bunche met l'accent sur les dynamiques du changement et de la modernisation, et il privilégie ainsi l'idée qu'il n'est, «du point de vue du développement social ", aucune différence entre l'Afrique et le monde occidental (ibid. $1934: 128,1936 \mathrm{c}: 63$ ). Bien évidemment, son point de vue est imbu de marxisme et il n'est donc guère original pour son époque, mais il parvient tout de même à dépasser les approches naturalistes ou strictement nationales de la question raciale en défendant une perspective résolument historique et globale.

« For either a White or a Black man it is scarcely more pleasant to be exploited and oppressed by privileged members of one's own race than by members of some other race. [...] Race issues but tend to merge into class issues. [...] The titanic conflicts of the future will be the product of the uncompromising struggle between those who have and those who have not. These conflicts now wage with all groups, racial and national. Race war then will be merely a side-show to the gigantic class war which will be waged in the big tent we call the world ».(Bunche 1936c : 95-96)

Dans le prolongement de ces premiers travaux, Bunche dépose en 1936 un nouveau projet de recherche auprès du Social Science Research Council (SSRC) ${ }^{31}$, qui vise désormais à étudier « l'impact du régime colonial et de la culture occidentale sur les 
Africains, du point de vue des Africains eux-mêmes » (Edgar 1992:11, 37 ; Urquhart 1993 : 62). Sur les conseils du sociologue Donald Young, alors directeur du SSRC et avec l'appui crucial de Melville Herskovits, qu'il connaissait depuis 1932, Bunche donne un tour résolument plus épistémologique que politique à son projet, puisqu'il demande à se former auprès de différents anthropologues sur leurs manières respectives d'étudier les contacts culturels. Il obtient ainsi un financement sur deux ans, avec pour feuille de route une formation postdoctorale auprès d'Herskovits à Northwestern University, de Malinowski à la London School of Economics, et d'Isaac Shapira (1905-2003) à l'Université du Cap, en Afrique du Sud. Bunche s'engage en outre à mener une enquête de terrain en Afrique de l'Est, puis à se rendre en Indonésie pour y étudier les spécificités de la politique coloniale hollandaise. Il devient ainsi le premier - et restera jusqu'aux années 1950 le seul - Afro-américain à voir ses recherches africanistes financées par les fondations (Edgar 1992 : 11-12; Holloway 2002 : 180).

Après quatre mois passés aux côtés d'Herskovits, à la fin de l'année 1936, Bunche séjourne donc à Londres, où il rencontre et fréquente Jomo Kenyatta (1893-1978) dans le séminaire de Malinowski, mais également de nombreux intellectuels panafricanistes comme C.R. L. James (1901-1989) et George Padmore (1902-1959) (Edgar 1992: 14; Urquhart 1993 : 66-67). Puis il se rend à la Hague, aux Pays-Bas, à la fin du mois de juillet 1937, avant d'embarquer fin septembre pour l'Afrique du Sud, où il réside d'abord un mois auprès de Shapira à Capetown pour visiter ensuite Pretoria, Bloemfontein, le Basutoland et Durban, du 28 septembre 1937 au $1^{\mathrm{er}}$ janvier 1938. Bunche va ensuite au Kenya et en Ouganda, et en avril 1938 il fait route vers Singapour, d'où il se rend à Java et Bali pour finalement rejoindre les États-Unis depuis Hong-Kong en juillet. Durant ce long périple - qui fait évidemment penser au « schème colossal » jadis élaboré par Alain Locke en 1910 - Bunche tient plusieurs journaux, personnels et scientifiques, où il consigne ses multiples impressions et observations. Il entreprend, en 1939, d'en tirer une monographie, financée par le SSRC et qui proposerait « une analyse $\mathrm{du}$ statut politique, économique et social des peuples non européens en Afrique du Sud », selon le plan suivant :

«An historical survey, the South African government and its color policy, labor problems, social controls, significant forces in white South Africa, what European and "non-European" South Africans think, "non-European" political and protest organizations, and a comparison and contrast of the American and South African experiences in race relations ».(Edgar $1992: 314$ )

Dans cette perspective, Bunche envisage d'effectuer un séjour dans le sud des ÉtatsUnis, afin d'y mener une enquête comparative de terrain. Mais d'abord ralenti par ses imposantes charges pédagogiques et administratives, de retour à Howard, il doit bientôt se résoudre à un autre agenda : sur la recommandation de Young, curateur de la Carnegie Corporation en plus de ses fonctions au SSRC, le jeune chercheur est en effet recruté par Gunnar Myrdal pour l'assister dans sa grande enquête sur le Nègre américain. C'est donc aux côtés de ce dernier qu'il effectuera finalement son voyage dans le Sud, et qu'il tirera parti de ses expériences africaines pour rédiger en 1940 plusieurs memoranda: A Brief and Tentative Analysis of Negro Leadership; Conceptions and Ideologies of the Negro Problem ; Ideologies, Tactics and Achievements of Negro Betterment and Interracial Organizations; The Political Status of the Negro in the Age of Franklin Delano Roosevelt. Largement recyclés dans l'ouvrage final de l'enquête Myrdal, An American Dilemma, certains de ces textes seront également publiés à titre posthume, à l'instar des notes de terrain sud-africaines qui avaient servi de laboratoire à nombre de leurs idées. 
La lecture de ces dernières est d'autant plus instructive que Bunche fut, avec l'anthropologue Eslanda Robeson (1896-1965), l'un des rares Afro-américains à pouvoir voyager dans cette Union de l'Afrique du Sud indépendante de l'Angleterre depuis 1910, et que ses recherches africanistes lui permirent ensuite de contribuer activement au déploiement de nouveaux paradigmes d'analyse sociale ${ }^{32}$.

Voulant étudier, ainsi qu'il le note le 7 novembre 1937, «comment une poignée de Blancs peut garder sous sa domination ces millions de Noirs» (Edgar 1992: 116), Bunche visite des villes et des villages, des fermes et des mines, des églises et des écoles, des universités et des congrès politiques, multipliant les entretiens, les portraits et les descriptions. Il s'intéresse aux techniques de la domination, au travers notamment de la politique foncière (Native Land Act, 1913) et de la politique urbaine (Native Urban Areas Act, 1923), qui ont permis d'instaurer la ségrégation en même temps qu'elles dépouillaient les Africains de leurs terres et de tout accès à la propriété. Il étudie aussi l'exercice du pouvoir, avec la privation des droits civiques (Edgar 1992:130,190) et la mise en place compensatoire du Native Representative Council, un collège restreint et mixte, à vocation purement consultative, majoritairement composé de Blancs et de quelques Noirs, et qui existera jusqu'en 1950. Bunche s'intéresse alors aux organisations politiques des Noirs sud-africains, depuis l'African National Congress (qui fête alors ses 25 ans d'existence sous la direction de son fondateur Pixley Isaka Seme, l'ami d'Alain Locke depuis Oxford) jusqu'à la National Liberation League, fondée en 1935 par Cissie Gool (1900-1963), ou encore la All African Association, créée la même année par Davidson Tengo Jabavu (1885-1959). S'il s'avoue déçu par les leaders de couleur, davantage soucieux de leurs minces avantages et de leur respectabilité que portés à une véritable contestation politique, Bunche dévoile les causes de leur inertie. Il examine en effet quelles divisions sociales (Edgar 1992:29, 66, 75, 82) et quels complexes psychologiques (ibid. : 131, 135, 171) sont induits par la hiérarchie raciale ; il relève les stratégies escapistes ou désespérément assimilationnistes qui en découlent, pour constater en dernier ressort la scission toujours croissante entre les élites et les masses. Pour sa part Bunche ne cesse, dans ses interventions auprès d'étudiants ou de militants politiques, d'insister sur la nécessité d'être fier, entreprenant et ambitieux, et de citer à cet effet des exemples afro-américains de médecins, juristes, universitaires ou chercheurs. En soulignant quel rôle moteur incombe à l'élite intellectuelle et politique dans la défense et l'émancipation des minorités opprimées, il reste fidèle à l'engagement des New Negroes, mais ses propos préfigurent également les conférences que prononcera, quelques années plus tard, Alain Locke en Haïti (Edgar 1992 : 23, 111, 131 ; Locke 2009 : 93-117). À l'instar de son collègue philosophe, Bunche multiplie les analogies entre les situations africaines et afro-américaines - dans ses carnets de voyage comme dans des lettres privées. "J'ai le mal du pays ", écrit-il par exemple à Herskovits le 7 octobre 1937, "chaque fois que je vois le signe: "réservé aux Européens" » (Edgar 1992: 27); tandis qu'à son retour aux États-Unis, il envoie cette lettre du 27 décembre 1938 à Cissie Gool, alors présidente de la National Liberation League :

«I am amazed at the number of close parallels there are between your situation and ours here. The pattern of racial (within the broader class pattern, of course) persecution and exploitation is universal. [...] I do think it is important for us to establish some close connecting links with you down there. Both the international Committee on African Affairs and the NAACP would very much like to keep abreast of the activities of the Liberation League and other organizations working on behalf of the non-European peoples of South Africa ».(cité dans Urquhart $1993: 80)^{33}$ 
39 À la lecture des analyses et des réflexions consignées dans les courriers, les carnets et les mémoires de recherche de Ralph Bunche, on devine quel rôle majeur il eût pu jouer dans le programme d'études africaines que projetait Alain Locke vers la fin des années 1930. Mais, ainsi que nous l'avons déjà noté, ce dernier resta lettre morte et il faudra finalement attendre 1954 - soit l'année même de la mort de Locke - pour que la Fondation Ford accorde enfin un don à Howard University afin d'y créer un programme d'études africaines (Harris $1981: 16$ ).

Durant la Seconde Guerre mondiale, quand l'Afrique avait constitué un terrain majeur de la lutte entre les Alliés et les forces de l'Axe, les fondations avaient pu mesurer l'importance cruciale, pour les États-Unis, d'une connaissance des réalités africaines. Le Social Science Research Council, l'American Council of Learned Societies ${ }^{34}$ et le National Research Council ${ }^{35}$ s'associèrent pour former l'Ethnogeographic Board, un comité essentiellement financé par la Carnegie Corporation et la Rockefeller Foundation et destiné à fournir des informations stratégiques au gouvernement américain (Gershenhorn 2004: 178). Les études d'aires culturelles (area studies) furent donc encouragées pour des motifs géopolitiques, et dans le contexte de l'après-guerre, qui devint rapidement celui de la guerre froide, les fondations commencèrent à financer les premiers programmes d'études africaines, d'abord à Fisk University et à l'Université de Pennsylvanie, de 1943 à 1947, puis à l'Université de Northwestern à compter de $1948^{36}$. Pour créer son propre programme, Herskovits convainquit la fondation Rockefeller que les universités noires américaines n'étaient pas les mieux placées, et qu'une partie des fonds traditionnellement alloués à l'Institut international des langues et des civilisations africaines de Londres pouvaient être redéployés pour former des chercheurs dans les universités américaines (Gershenhorn 2004 : 180,184). Dix ans plus tard, Herskovits devait fonder l'African Studies Association (ASA) afin de coordonner le développement des études africaines aux États-Unis. Entre-temps, l'Université d'Howard s'était à son tour dotée d'un programme interdisciplinaire dont la présentation générale fut publiée en 1960 dans le Bulletin de l'ASA aux côtés des autres programmes africanistes américains (universités de Boston, de Californie, de Chicago, de Columbia, de Duke, de Duquesne, de Northwestern, de Roosevelt, de Stanford et de Yale, en sus de la Hartford Seminary Foundation et de la New School for Social Research). Les objectifs déclarés étaient de fournir une formation aux étudiants africains et afro-américains d'Howard sur «la place de l'Afrique dans le monde moderne » et sur "ses problèmes économiques, sociaux, politiques ». Pour cela, en sus des cours de langues africaines (en particulier le swahili et le yoruba), les enseignements portaient sur "la géographie humaine en Afrique, les peuples et les cultures d'Afrique, l'histoire de la colonisation, l'administration des territoires africains, l'économie dans le monde moderne, l'impact des civilisations occidentales en Afrique, et l'histoire des anciennes civilisations en Afrique tropicale». Le comité en charge de ce programme interdisciplinaire était dirigé par le sociologue Ezra Franklin Frazier, et composé des historiens Rayford W. Logan et Leo Hansberry, du géographe Anthony S. Reyner, de l'anthropologue Mark Watkins, et de l'économiste Bernard Blankenheimer (African Studies Association 1960 : 35-36).

41 Ce programme d'études africaines portait certes l'empreinte des projets élaborés par Alain Locke dans les années 1920 et 1930, mais il était surtout profondément marqué par la nouvelle donne des area studies: la préséance accordée à l'histoire, à la géographie, à l'économie sur l'étude de la littérature ou de la pensée africaines révélait 
en effet une fonction clairement géopolitique. Sous la tutelle de Frazier, ardent défenseur de l'intégration noire américaine et, de ce fait, farouche critique des travaux d'Herskovits sur les survivances africaines, la perspective culturaliste et revivaliste du mouvement "Nouveau Nègre" n'était plus de mise, et Leo Hansberry se voyait également attribuer une place secondaire. Rayford W. Logan assurait les cours sur l'histoire et l'administration coloniales en Afrique: ses travaux sur le système des mandats africains (Logan 1928, 1945, 1948) avaient certes anticipé et même prolongé ceux de Bunche, mais par-delà son implication dans le mouvement panafricaniste des années 1920 aux années 1940, Logan n'avait pas l'expérience ni l'aptitude à s'identifier au point de vue africain de son collègue politologue et anthropologue. Il n'est revenu à Paris qu'en 1951, sur une bourse Fullbright, pour y étudier le nouveau système colonial français dans le cadre de l'Union française (Janken 1993: 196, 217), et lorsque le Département d'État l'envoie, deux ans plus tard, poursuivre ses recherches sur cette question en Afrique de l'Ouest, Logan prend surtout conscience de la distance qui le sépare des autochtones :

« My trip to West Africa in the summer of 1953 convinced me that the more I knew about "the Dark Continent" the less likely I would participate in a revival of "Black Nationalism" [...]. I am more interested in my Wittingham ancestry than in my African forbearers [...]. When I landed at Dakar, I felt no great kinship to Africa as did Du Bois when he arrived in Monrovia ».(Logan cité dans Janken 1993 : 197)

À l'instar des Européens, Logan était imbu de la supériorité de sa culture occidentale mais il partageait aussi, comme de nombreux panafricanistes, leur vision plutôt paternaliste et condescendante de l'Afrique : dans son esprit, les Africains restaient loin de pouvoir se gouverner eux-mêmes, et le principe de la tutelle conservait toute sa pertinence au sortir de la guerre. Dans un « Memorandum on a Proposed New Mandate System ", soumis en décembre 1942 à Benjamin Gerig (1894-1976), un diplomate américain qui avait siégé à la Commission des mandats de la Société des Nations, et qui travaillait désormais pour le Département d'État à l'élaboration d'un nouveau système de curatelle internationale (international trusteeship), Logan défendait la création d'une nouvelle Commission permanente des mandats où siégeraient des puissances non coloniales comme le Brésil, Haïti, le Liberia ou l'Abyssinie, afin de superviser le développement des peuples et des territoires colonisés en Afrique jusqu'à leur complète indépendance, grâce en particulier à l'instauration d'une école internationale d'administration coloniale (Janken 1993 : 169-174). Les options de Logan et son rôle de consultant auprès du Département d'État nous renseignent en creux sur l'étonnante absence de Ralph Bunche dans la mise en place des études africaines à Howard, dans les années 1950 : c'est que le développement historique des areas studies avait de fait ouvert de nouvelles carrières aux chercheurs spécialisés dans les études politiques ou anthropologiques de l'Afrique. La Carnegie Foundation for the Advancement of Teaching estimait ainsi, en 1947, qu'il y avait en matière d'aires culturelles plus de besoins que de spécialistes effectifs pour alimenter les corps diplomatiques et les institutions internationales (Powers 1955: 85). C'est pour ces raisons précises qu'en septembre 1941, son expertise africaine vaut à Ralph Bunche d'être recruté par l'office of Strategic Services - ancêtre de la Central Intelligence Agency - en qualité de senior social-science analyst avec pour mission d'élaborer diverses synthèses sur les événements, les attitudes dominantes et les problèmes quotidiens des populations dans les empires britannique et français en Afrique (Urquhart 1993 : 102). Sa connaissance du système des mandats et de ses insuffisances le conduit à participer, à la même 
époque, aux travaux du Committee on Africa, the War and Peace Aims, financé par le Phelps Stokes Fund ${ }^{37}$. L'ouvrage qui parait (The Atlantic Charter and Africa from an American Standpoint, 1942) défend clairement « la nécessité d'avancer rapidement vers une participation plus large et responsable des peuples colonisés au gouvernement de leur pays", ainsi que "le but ultime d'un gouvernement autonome pour toutes les colonies, y compris en Afrique» (Locke 1942a : 527). En attendant et pour atteindre cette perspective,

"The Mandate ideal of the vital importance of native rights, welfare, and development should be applied in all African territory controlled by European powers and should be adopted by the independent African States ».(ibid.)

Ces vues trouveront un large écho dans « Color, Unfinished Business of Democracy » où, en sus de l'introduction rédigée par Locke, trois articles sont consacrés à la situation africaine. Le premier rappelle l'importance des efforts missionnaires américains pour l'éducation en Afrique, ainsi que les principales propositions du « Comité sur l'Afrique, la guerre et les objectifs de la paix » (Ross 1942); le deuxième souligne les exigences démocratiques des peuples africains, en raison même de leur engagement dans le conflit mondial (Mbadiwe 1942), tandis que le troisième expose l'action de Félix Eboué (1884-1944), rallié à la France Libre et gouverneur de l'Afrique équatoriale française, dans ses efforts pour réformer en profondeur la politique coloniale de la France (Kaskeline 1942). Une hésitation demeure pourtant entre, d'une part, le principe acquis de la légitimité des revendications nationalistes et indépendantistes et, d'autre part, le rêve renouvelé d'une réforme des systèmes coloniaux et leur remplacement par un véritable internationalisme (Locke 2009 : 43-45). Or c'est précisément cette tension qui motivera, par la suite, toute la carrière internationale de Ralph Bunche. Après avoir été recruté par Benjamin Gerig au Département d'État en 1944, pour collaborer à l'élaboration du plan de paix sur les questions coloniales, il sera transféré auprès des Nations Unies pour participer à l'élaboration de leur Charte. À force de patience, de débats et de compromis, le politologue noir américain deviendra un des principaux rédacteurs de l'article XI ("Déclaration relative aux territoires non autonomes»), lequel promeut officiellement le principe de la curatelle dans une visée en dernier ressort indépendantiste ${ }^{38}$. En 1950, Bunche obtient le Prix Nobel de la Paix pour avoir négocié le premier accord de paix entre Israël et un certain nombre de pays arabes. Dans son discours de réception à Oslo, il revient sur la nécessité d'« accélérer la liquidation du colonialisme et [de] tendre une main amie vers les peuples qui s'échinent sous le lourd fardeau d'une indépendance récemment gagnée, ou qui tout simplement y aspirent » en leur fournissant toute l'aide nécessaire: des fonds, des vivres, des équipements et une assistance technique (Bunche 1950 cité dans Rivlin 1990 : 233). Convaincu que la cause des peuples colonisés constituait la voie historique pour combler enfin le grand écart entre les idéaux démocratiques au nom desquels la Seconde Guerre mondiale avait été menée, et leurs dévoiements dans les réalités coloniales (Urquhart 1993: 135), il œuvra constamment à garantir l'autonomie et l'émancipation africaines, jusque dans la crise de l'indépendance congolaise qui devait, en 1960, tragiquement opposer le gouvernement de Patrice Lumumba (1925-1961) aux troupes militaires belges et aux forces sécessionnistes de Moïse Tshombé (1919-1969) au Katanga (ibid. : 299-335 ; Hill \& Keller 2010). 

forment un singulier binôme dont les polarités manifestent très bien les multiples tensions installées au cœur du projet afro-américain des African Studies. Des mobiles idéologiques (la fierté compensatoire, l'ancrage nationaliste, le projet identitaire) parasitent en effet un dessein strictement politique (la conquête de l'égalité juridique et sociale), tandis que des enjeux scientifiques (la critique de l'évolutionnisme) entrent clairement en porte-à-faux avec des perspectives humanitaristes (l'aide au développement économique, social, intellectuel et moral du monde noir). Dans ces postulations contradictoires résidait le rapport fondamentalement ambivalent des intellectuels noirs américains à l'Afrique coloniale, vue tout à la fois comme trop 
étrange pour être semblable, et trop familière pour rester tout à fait étrangère. Au-delà des liens imaginaires ou sentimentaux qui les attachaient à elle, leur grandeur fut pour leur époque d'en appréhender les réalités en termes «communs ». Mais on peut aussi se demander, rétrospectivement, si leurs postulations contradictoires n'ont pas, en amont, préparé les divergences actuelles entre afrocentrisme et africanisme, ou entre African Studies et Black Studies dans le monde académique américain.

\section{BIBLIOGRAPHIE}

AFRICAN STUDIES ASSOCIATION, 1960 «African Studies in the United States », African Studies Bulletin, 3 (1) : 31-40.

AMSELLE, J.-L., 2001 Branchements. Anthropologie de l'universalité des cultures, Paris, Flammarion. AMSELLE, J.-L. \& SIBEUD, E. (dir.), 1998 Maurice Delafosse, entre orientalisme et ethnographie : l'itinéraire d'un africaniste (1870-1926), Paris, Maisonneuve \& Larose.

ANDERSON, J., 1980 «Philanthropic Control Over Private Black Higher Education », in R. ARNOVE (ed.) : Philanthropy and Cultural Imperialism : The Foundations at Home and Abroad, Boston, G. K. Hall : 147-178.

APTHEKER, H., 1977 A Documentary History of the Negro People in the United States, 1910-1932, Secausus, The Citadel Press.

ASSELINEAU, R., 1966 Robert Frost, Paris, Seghers (« Poètes d'aujourd'hui »).

BOAHEN, A., 1987 African Perspectives on Colonialism, Baltimore, John Hopkins Press.

BOAS, F., 1922 [1911] The Mind of Primitive Man, New York, The MacMillan Company.

BUNCHE, R., 1934 French Administration in Togoland and Dahomey, Ph. D. Dissertation, Cambridge (Mass.), Harvard University.

-, 1936a « French and British Imperialism in West Africa », Journal of Negro History, 21 (1) : 31-46.

,$- 1936 \mathrm{~b}$ « Education in Black and White », Journal of Negro Education, 5 (3) : 351-358.

-, 1936c A World View of Race, Washington D.C., The Associates in Negro Folk Education.

-, 1936a 1937 « Culture Conflict in South Africa : Review of Monica Hunter's Reaction to

Conquest », Journal of Negro Education, 6 (4) : 639-642.

-, 1936a 1939 « Dahomean Culture. Review of Dahomey, by Melville Herskovits », Journal of Negro Education, 8 (2) : 209-212.

-, 1936a 1954 « The Passing of Alain Leroy Locke », Phylon, 15 (3) : 243.

-, 1936a 1973 The Political Status of the Negro in the Age of Franklin Delano Roosevelt, Chicago, Chicago University Press.

-, 1936a 1990 « Some Reflections on Peace in Our Time, Nobel Peace Prize Lecture, Oslo, Norway, 11 December 1950 », in B. RIVLIN, (ed.), Ralph Bunche, The Man and His Times, New York-London, Holmes \& Meier : 225-235. 
DEWITTE, P., 1985 Les Mouvements nègres en France, 1919-1939, Paris, L'Harmattan.

DU BoIS, W. E. B., 1915 The Negro, New York, Henry Holt \& Company.

-, 1936a 1919 « Returning Soldiers », The Crisis, 18 (5) : 7-14.

DURPAIRE, F., 2005 Les États-Unis ont-ils décolonisé l'Afrique noire francophone ?, Paris, L'Harmattan.

EDGAR, R. (ed.), 1992 An African-American in South Africa, The Travel Notes of Ralph J. Bunche, 28 september 1937-1 january 1938, Athens, Ohio University Press.

VON ESCHEN, P., 1997 Race Against Empire, Black Americans and Anticolonialism, 1937-1957, Ithaca, Cornell University Press.

FINNEGAN, C., 2000 Social Welfare and Visual Politics : The Story of The Survey Graphic, (<http:// newdeal.feri.org/sg/essay.htm>).

FROST, R., 1995 [1916] « The Road Not Taken », in R. FROST (ed.), Collected Poems, Prose and Plays, New York, Library of America : 103.

GERSHENHORN, J., 2004 Melville J. Herskovits and the Racial Politics of Knowledge, Lincoln, University of Nebraska Press.

GOGGIN, J., 1983 « Countering White Racist Scholarship : Carter G. Woodson and the Journal of Negro History ", The Journal of Negro History, 68 (4) : 355-375.

-, 1936a 1993 Carter G. Woodson, A Lige in Black History, Bâton Rouge-London, Louisiana State University Press.

HANSBERRY, L., 1921 « The Material Culture of Ancient Nigeria », Journal of Negro History, 6 (2) : 261-295.

-, 1936a 1981 Pillars in Ethiopian History, African History Notebook, Vol. 1., Washington D. C., Howard University Press.

HARRIS, J. E., 1981 « William Leo Hansberry, 1894-1965, Profile of a Pioneer Africanist », in L. HANSBERRY (ed.), Pillars in Ethiopian History, African History Notebook, op. cit. : 3-30.

HARRIS, L. \& MOLESWORTH, C., 2008 Alain L. Locke, Biography of a Philosopher, Chicago-London, University of Chicago Press.

HARRIS, R., 1982 « Segregation and Scholarship : The American Council of Learned Societies' Committee on Negro Studies, 1941-1950», Journal of Black Studies, 12 (1) : 315-331.

HARRIS, L. (ed.), 1989 The Philosophy of Alain Locke, Harlem Renaissance and Beyond, Philadelphia, Temple University Press.

HENRY, C. P., 1990 " Civil Rights and National Security : The Case of Ralph Bunche », in B. RIVLIN (ed.), Ralph Bunche, The Man and His Times, New York-London, Holmes \& Meier : 50-66.

HERSKOVITS, M., 1925a « Preliminary Observations in a Study of Negro-White Crossing ", Opportunity, 3 (3) : 69-74.

,$- 1925 b$ « The Negro's Americanism », in A. LOCKE (ed.), The New Negro, An Interpretation, New York, Albert \& Charles Boni : 353-360.

-, 1936a 1926 « Does the Negro Know His Father? A Study in Negro Genealogies », Opportunity, (10) : 306-310.

-, 1958 [1941] The Myth of the Negro Past, Boston, The Beacon Press. 
HILL, R. A. \& KELLER, E. J. (eds.), 2010 Trustee for the Human Community : Ralph J. Bunche, The United Nations and the Decolonization of Africa, Paris, Maisonneuve \& Larose.

HINE, D. C., 1986 « Carter G. Woodson, White Philanthropy and Negro Historiography », The History Teacher, 19 (3) : 405-425.

HollowaY, J. S., 2002 Confronting the Veil : Abram Harris Jr, E. Franklin Frazier, and Ralph Bunche, 1919-1941, Chapell Hill-London, The University of North Carolina Press.

-, 2005 A Brief and Tentative Analysis of Negro Leadership, by Ralph J. Bunche, New York, New York University Press.

JACKSON, W., 1986 « Melville Herskovits and the Search for Afro-American Culture », in G. STOCKING (ed.), Malinowski, Rivers, Benedict and Others, Essays on Culture and Personality, Madison, University of Wisconsin Press : 95-126.

JANKEN, R. K., 1993 Rayford W. Logan and the Dilemma of the African American Intellectual, Amherst, University of Massachussetts Press.

JONES, T. J., 1922 Education in Africa, New York, Phelps-Stokes Fund.

KASKELINE, E., 1942 « Félix Eboué and the Fighting French », in A. LOCKE (ed.), « Color, the Unfinished Business of Democracy », The Survey Graphic, 31 (11) : 522-523, 548-550.

KILSON, M., 1990 " Ralph Bunche's Analytical Perspective on African Development », in B. RIVLIN (ed.), Ralph Bunche, The Man and His Times, New York-London, Holmes \& Meier : 83-95.

DE L’ESTOILE, B., 1997 « “Africanisme” et “Africanism”, esquisse de comparaison francobritannique », in A. PIRIOU \& E. SIBEUD (dir.), L’Africanisme en questions, Paris, EHESS, Centre d'études africaines (« Dossiers Africains ») : 19-42.

LEWIS, D. L., 1993 W. E. B. Du Bois, Biography of a Race, 1868-1919, New York, Henry Holt \& Company. -, 2000 W. E. B. Du Bois, the Fight for Equality and the American Century, 1919-1963, New York, Henry Holt \& Company.

LOCKE, A., 1923a « The Colonial Literature of France », Opportunity, 1 (11) : 331-335.

-, 1923b « Roland Hayes : An Appreciation », Opportunity, 1 (12) : 356-358.

,$- 1924 a$ « Black Watch on the Rhine », Opportunity, 2 (1) : 6-9.

,$- 1924 b$ « Apropos of Africa », Opportunity, 2 (2) : 37-40.

-, 1924c « Impressions of Luxor », The Howard Alumnus, 2 (4) : 74-78.

,$- 1924 \mathrm{~d}$ « As Others See Us : Review of La Question des Noirs aux États-Unis, by Frank

L. Schoell », Opportunity, 2 (4) : 109-110.

-, 1924e « A Note on African Art », Opportunity, 2 (5) : 134-138.

-, $1924 \mathrm{f}$ « French Colonial Policy (Open Letter to René Maran) », Opportunity, 2 (9) : 261-263.

-, 1924g « La jeune poésie africo-américaine », Les Continents, $8: 2$.

-, 1925a « Backstage on English Imperialism : A Review of The Story of My Life, by Sir Harry

H. Johnston », Opportunity, 3 (4) : 112-114.

-, 1925b « Harlem, Mecca of the New Negro », The Survey Graphic, 6 (3) : 621-725.

-, 1925c « The Art of Auguste Mambour », Opportunity, 3 (8) : 240-241, 252.

,$- 1925 \mathrm{~d}$ « More of the Negro in Art », Opportunity, 3 (12) : 363-365. 
,$- 1925 \mathrm{e}$ « Colonial Commonsense : Review of Kenya, by Norman Leys », The Survey Graphic, 6

(12) : 380-381.

-, 1925f The New Negro, An Interpretation, New York, Albert \& Charles Boni.

-, 1927a « A Collection of Congo Art », The Arts, 11 (2) : 60-70.

,$- 1927 \mathrm{~b}$ « Arts Lessons from the Congo : Blondiau Theatre Arts Collection », The Survey Graphic, $57,8(2): 587-589$.

-, 1928a « Memorandum on Department of African Studies at Howard University », HU, MSRC, ALP, 164-163/19.

-, 1928b « The Mandate System : A New Code of Empire », HU, MSRC, ALP, 164-116/16.

-, 1931 « Le Nègre Nouveau », traduit en français par Louis Guilloux, Europe, 102 : 289-300.

-, 1932 «We Turn to Prose : A Retrospective Review of the Literature of the Negro for 1931 », Opportunity, 10 (2) : 40-44.

-, 1936a The Negro and His Music, Washington D.C., Associates in Negro Folk Education.

-, 1936b Negro Art: Past and Present, Washington D.C., Associates in Negro Folk Education.

-, 1942a « Color, the Unfinished Business of Democracy », The Survey Graphic, 31 (11) : 453-575.

-, 1942b When Peoples Meet : A Study in Race and Culture Contacts, New York, Progressive Education Association.

,$- 1942 \mathrm{c}$ « Who and What is "Negro" ? », Opportunity, 20 (1-2) : 36-41, 83-87.

-, 1950 « Wisdom De Profundis : The Literature of the Negro, 1949 », Phylon, 11 : 5-14, 171-175.

-, 1951 « L'apport intellectuel et culturel du Noir américain », Études américaines, 29 : 3-6.

-, 1992 Race Contacts and Interracial Relations, Washington D.C., Howard University Press.

-, 2005 « Peace between Black and White in the United States », World Order, 36 (3) : 42-45; HU, MSRC, ALP, 164-123/19.

-, 2009 Le rôle du Nègre dans la culture des Amériques, Paris, L’Harmattan (« Autrement Mêmes »). LOGAN, R. W., 1928 " The Operation of the Mandate System in Africa », The Journal of Negro History, $13(4): 423-477$.

-, 1942 The Operation of the Mandate System in Africa, 1919-1927 : with an Introduction on the Problem of the Mandates in the Post-War World, New York, The Foundation Publishers.

-, 1945 The Senate and the Versailles Mandate System, Washington D.C., Minorities Publishers.

-, 1948 The African Mandates in World Politics, Washington D.C., Public Affairs Press.

MANGEON, A., 2004 Lumières Noires, Discours Marron, indiscipline et transformations du savoir chez les écrivains noirs américains et africains, itinéraires croisés d'Alain Leroy Locke, V. Y. Mudimbe et de leurs contemporains, Thèse de doctorat, Cergy, Université de Cergy-Pontoise.

MBADIWE, K. O., 1942 « Africa's Hope for Democracy », in A. LOCKE (ed.), « Color, the Unfinished Business of Democracy », op. cit. : 519-521, 550-552.

MYRDAL, G., 1944 An American Dilemma, The Negro Problem and Modern Democracy, New York-London, Harper \& Brothers.

PADMORE, G., 1960 Panafricanisme ou Communisme? La prochaine lutte pour l'Afrique (trad. de l'anglais par Thomas Diop), Paris, Présence Africaine. 
PARK, R. E., 2008 Le Journaliste et le Sociologue, textes réunis et commentés par Géraldine Muhlmann et Edwy Plenel (trad. de l'anglais par Cécile Deniard), Paris, Éditions du Seuil.

PIRIOU, A. \& SIBEUD, E. (dir.), 1997 L'Africanisme en questions, Paris, EHESS, Centre d'études africaines (« Dossiers Africains »).

POWERS, M. K., 1955 « Areas Studies », The Journal of Higher Education, 26 (2) : 82-113.

RIVLIN, B. (ed.), 1990 Ralph Bunche, The Man and His Times, New York-London, Holmes \& Meier.

ROSS, E., 1942 « America and Africa », in A. LOCKE (ed.), « Color, the Unfinished Business of

Democracy », op. cit. : 524-527, 574-575.

SENGHOR, L., 1927 « La question nègre devant le Congrès de Bruxelles, discours de Senghor ", La

Voix des Nègres, 1 (bis) : 1 .

SIBEUD, E., 2002 Une science impériale pour l'Afrique? La construction des savoirs africanistes en France, 1878-1930, Paris, EHESS.

SMITH, M., 1994 Social Science in the Crucible: The American Debate over Objectivity and Purpose, 1918-1941, Durham, Duke University Press.

STEWART, J., 1979 A Biography of Alain Locke, Philosopher of the Harlem Renaissance, 1886-1930, Ph. D. Dissertation, New Haven (Connect.), Yale University.

-, 1983 The Critical Temper of Alain Locke : A Selection of His Essays on Art and Culture, New YorkLondon, Garland Publishing Inc.

STOCKING, G., 1985 «Philanthropoids and Vanishing Cultures : Rockefeller Funding and the End of the Museum Era in Anglo-American Anthropology ", in G. STOCKING (ed.), Objects and Others : Essays on Museum and Material Culture, Madison, University of Wisconsin Press : 112-145.

URQUHART, B., 1993 Ralph Bunche, An American Odyssey, New York-London, Norton \& Company.

\section{NOTES}

1. «Worlds of Color : The Negro Mind Reaches Out » (LOCKE 1925f : 385-414).

2. J'emprunte cette formule au poète américain Robert Frost (1874-1963). Cité en épigraphe, son poème «The Road Not Taken» est traduit en français par «La voie ignorée » ou par « La route non prise » (ASSELINEAU 1966).

3. «I admit I was fairly staggered by the "colossal" scheme - it made my poor head swim » (Mary Locke à Alain Locke, Howard University, Moorland-Spingarn Research Center, Alain Locke Papers, box 164-58, folder 17 ; (désormais abrégé HU, MSRC, ALP, $\mathrm{XXX}-\mathrm{XX} / \mathrm{X})$.

4. Voir notamment l'itinéraire de Robert Ezra Park (1864-1944) : d'abord journaliste, il travailla aux côtés de Booker T. Washington afin de devenir un sociologue de renom sur la question des relations raciales, et de fonder l'école de Chicago (PARK 2008).

5. "The scheme seems to have assume [sic] quite some proportions, and I have high hopes not only of making it a success as a personal and journalistic venture, but as furnishing some material of permanent value towards the comparative study of the race problem » (Lettre d'Alain Locke à Booker T. Washington, en date du 15 juin 1910, 
Archives Booker T. Washington, 408-Li, Bibliothèque du Congrès, Washington D. C., cité par STEWART 1979 : 171).

6. HU, MSRC, ALP, 164-127/33.

7. Le financier français Albert Kahn (1860-1940) avait en 1910 créé cette bourse de voyages afin d'encourager le développement du cosmopolitisme. Cette année-là, seuls deux candidats sur dix furent retenus.

8. Issu de la coordination de plusieurs sociétés de charité urbaines aux États-Unis, The Survey (1912-1952) était dirigé par le journaliste Paul U. Kellogg (1879-1958), dont l'étude approfondie sur les conditions de vie à Pittsburgh (The Pittsburgh Survey, 1910-1914) s'était imposée comme un modèle d'intervention de la recherche sociologique dans la réforme sociale. Pour une histoire détaillée du Survey, voir C. FINNEGAN (2000). Un grand nombre d'articles de ce " magazine d'interprétation sociale » sont également disponibles en ligne à l'adresse suivante : <http://xroads.virginia.edu/ $\sim$ MA01/Davis/survey/home.html>. La revue Opportunity fut créée en 1923 par le sociologue noir américain Charles S. Johnson (1893-1950) qui avait étudié avec Robert Park à l'Université de Chicago ; elle servait de tribune à la National Urban League, une association créée en 1911 pour aider les immigrants noirs des États ruraux du Sud à s'implanter dans les villes industrielles du Nord.

9. HU, MSRC, ALP, 164-127/6, p. 9 (« Separation or Fusion »).

10. HU, MSRC, ALP, 164-24/17.

11. Maurice Delafosse à Alain Locke, lettre en date du 13 mai 1924, HU, MSRC, ALP, 164-24/17.

12. Sur ce licenciement, voir HARRIS \& MOLESWORTH (2008: 175-176). Président (blanc) de l'Université d'Howard, James Durkee (1866-1951) avait déjà obtenu, en 1920, la démission de l'historien noir américain Carter G. Woodson (1875-1950), lequel avait en 1915 fondé The Association for the Study of Negro Life and History et dirigeait sa revue, The Journal of Negro History. Leo Hansberry fut recruté à Howard pour remplacer Woodson en 1922, et il orienta ses cours d'histoire africaine vers l'étude des civilisations antiques en Afrique (de l'Égypte à l'Éthiopie). Mais dès qu'il entreprit de défendre à son tour la création d'un Institut d'études africaines, il fut en butte à la même hostilité que Locke (HANSBERRY 1981 : 7-16). Le premier projet de Locke fut soumis au Board of Trustees de l'Université d'Howard le $1^{\text {er }}$ mai 1924 (HU, MSRC, ALP, 164-128/20).

13. HU, MSRC, ALP, 164-142/14 (« Notes on Africa »).

14. HU, MSRC, ALP, 164-116/16 ("The Mandate System : A New Code of Empire »).

15. HU, MSRC, ALP, 164-142/13 (« Colonial Administration and Policy in Africa »). Locke voulait visiter l'Afrique du Sud car celle-ci avait également hérité d'un mandat sur l'excolonie allemande appelée Sud-Ouest Africain, aujourd'hui la Namibie. Soumis à la Fondation Carnegie, ce projet sera rejeté par Charles T.Loram (1879-1940), un éducateur sud-africain (EDGAR 1992: 39). Dans les années 1930, Loram deviendra le directeur du Département d'étude des relations raciales à Yale University et il exercera auprès des fondations philanthropiques une influence très conservatrice et fondamentalement hostile aux chercheurs afro-américains (GERSHENHORN 2004: 152, 175).

16. Le premier Congrès panafricain se tient à Paris, du 19 au 21 février 1919 ; le deuxième a lieu à Londres, Bruxelles et Paris, du 28 août au 6 septembre 1921; le 
troisième a lieu en 1923 à Lisbonne (7-8 novembre) et à Londres ( $1^{\text {er }}-2$ décembre), et le quatrième à Harlem (New York), du 21 au 24 août 1927. Absent mais déjà mentionné durant le premier congrès, Logan participa activement aux trois suivants en assurant notamment la traduction des délibérations puis des résolutions de l'anglais au français, et vice-versa. Voir APTHEKER (1977 : 248-252, 335-347, 430-432, 544-548) et JANKEN (1993 : 49-57).

17. "Faculty committee Memorandum on a proposed "Institute of African Studies" ", HU, MSRC, ALP, 164-128/20, p. 1.

18. Les cours d'anthropologie mentionnés par Locke sont "African Negro Economics and Customary Law », "Culture Strata and Culture Areas in Africa », " The Problem of Black and White in Africa ».

19. "The History of Ethiopia and Egypt in Ancient Times ", "West African Civilization in the Middle Ages ». Voir HARRIS (1981:6-7).

20. Davis avait étudié l'anthropologie sociale à Harvard puis à la London School of Economics. Il sera recruté par l'Université Dillard puis par l'Université de Chicago et se spécialisera dans l'étude des relations raciales et des modèles éducatifs.

21. "Criticism of the pseudo-scientific in even scientific interpretation: a) centers primarily in distortions of the theory of social evolution - using African cultures as the bottom ring, in a historical progression - the minimal values of the evolutionary scheme ; b) The assumption of the great homogeneity of African cultures ; c) The fallacy of the race interpretation of civilization and culture ; d) the false attribution of culture to race capacities or environmental factors. On the whole the advanced tendencies as they affect the interpretation of African culture may be summarized as follows : a) the introduction of the cultural strata and cultural area idea (Groebner \& Wissler); b) The cartographic method (Frobenius); c) The interpretation of material cultures by psychological interpreation (Rivers, Lowie); d) The comparative method; e) The independent variability of physical and cultural factors " («The Handicaps of African Study », HU, MSRC, ALP, 164-105/16, p. 2) ; « Any and all cultures must first be analyzed more historically and more objectively, and in terms of their intrinsic forms and values » ("The Transformation of African Studies », HU, MSRC, ALP, 164-128/16, p. 3). Voir également LOCKE (2009: 30-32).

22. «The Transformation of African Studies ", article non daté, 5 pages, HU MSRC ALP $164-1128 / 16$.

23. Dès 1925, Locke tempère l'assimilationnisme d'Herskovits en ajoutant la note suivante à son article, initialement paru dans "Harlem, Mecca of the New Negro ": "Looked at in its externals, Negro life, as reflected in Harlem registers a ready almost a feverishly rapid - assimilation of American patterns, what Mr Herskovits calls "complete acculturation". [...] Internally, perhaps, it is another matter. Does democracy require uniformity? [...] Social standards must be more or less uniform, but social expressions may be different. Old folkways may not persist, but they may leave a mental trace, subtly recorded in emotional temper and coloring social reactions " (LOCKE 1925b: 676). Sur l'influence de Locke sur Herskovits, voir JACKSON (1986: 100-109), GERSHENHORN (2004: 67), MANGEON (2004:330-339).

24. Sur les relations entre Woodson et les fondations, voir GOGGIN $(1983,1993)$ et LEWIS (2000: 191) ; sur Du Bois et son projet d'Encyclopedia Africana, longtemps retardé par le Phelps-Stockes Fund, en raison de l'hostilité d'Herskovits, et finalement initié au Ghana 
avec le soutien de Kwame Nkrumah, voir JACKSON (1986 : 116), LEWIS (2000:389, 427-429, 434-436, 566-568) et GERSHENHORN (2004: 148-156). Sur Locke et son projet avorté d'Encyclopedia Negro-Americana, voir HARRIS \& MOLESWORTH (2008: 127). Sur les projets d'Institut africain par Leo Hansberry, voir HARRIS (1981: 9-15). Pour une étude plus générale de l'incidence des fondations sur les projets de recherche afro-américains, voir ANDERSON (1980), HARRIS (1982), HINE (1986).

25. Sur le rôle consultatif d'Herskovits auprès des fondations, voir JACKSON (1986: 115-118), EDGAR (1992: 11, 12, 37), HOLLOWAY (2002 : 180). Sur ses propres difficultés à être financé dans les années 1920, puis sur son influence, son hostilité et son paternalisme à l'égard des intellectuels noirs, voir JANKEN (1993: 94-95), GERSHENHORN (2004 : 131-135, 143-145).

26. L'Avant-propos des responsables de la Carnegie Corporation au livre d'Herskovits est à cet égard tout à fait significatif : «We may concede that the greatest significance of the African heritage lies in the fact that most of it quickly and inevitably was lost before the ways of life of the dominant white man could be learned. Yet cultural differentials are so important in the social adjustment of different peoples to each other that the retention even of cultural fragments from Africa may introduce serious problems into Negro-white relations" (Harrison, Ogburn et Young cités dans HERSKOVITS $1958:$ X).

27. «Talented Tenth »: c'est ainsi qu'à la suite de Du Bois, l'élite intellectuelle afroaméricaine s'était autodésignée.

28. «As a subject race under white powers, [the Negro] must perforce interest himself in the methods of government and administration applied to him by these powers. It seems to me deplorable that the American Negro has seen fit to ignore these questions of international portent, and ostrich-like, to bury his head in the sands of relatively petty local problems» ("Colonial Administration and Policy in Africa », HU, MSRC, ALP, 164-142/13, p. 2).

29. On peut déjà en trouver un certain aveu dans la nécrologie que publia Bunche à la mort de Locke : « How much inspiration I have derived from his life and works I cannot possibly measure, but it has been great, for in my younger days, in the Twenties and Thirties, Alain Locke was a strong and unique beacon lighting an exciting new course for aspiring young Negroes » (BUNCHE 1954 : 243).

30. Cette critique sera par la suite réitérée à l'encontre d'Herskovits, soupçonné dans sa reconstruction historique de L'Ancien Royaume $d u$ Dahomey de n'avoir point suffisamment tenu compte des distorsions introduites par le colonialisme français, et d'avoir ainsi ignoré «la forêt impérialiste » derrière «l'arbre ethnologique » (BUNCHE 1939 : 212 ; EDGAR $1992: 12$ ).

31. Organisation de soutien à la recherche créée en 1923 à New York, et financée par les grandes fondations américaines, le gouvernement et des donateurs privés.

32. L'épouse du chanteur et comédien Paul Robeson étudia également à la London School of Economics et elle séjourna en Afrique du Sud en 1936. Elle publia dix ans plus tard un récit de voyage (An African Journey, 1945) largement remanié et coloré d'un tel parti pris marxiste qu'il perd de son authenticité et de sa spontanéité en comparaison avec les notes de Bunche.

33. L'International Committee on African Affairs fut fondé en 1937 par Max Yergan (1892-1975), un activiste afro-américain qui avait passé quinze ans en Afrique du Sud à 
la tête de la Young Men's Christian Association et qui s'était progressivement radicalisé. L'ICAA visait à faire connaître les réalités africaines et à aider les Africains venus étudier en Amérique. Ralph Bunche, Paul Robeson, le président d'Howard Mordecai Johnson, l'écrivain martiniquais René Maran comptent parmi ses premiers membres. Mais à l'exception de Robeson, tous prendront leurs distances quand Yergan s'affiliera au parti communiste américain. Devenue en 1942 le Council on African Affairs, l'association sera par la suite dirigée par Alphaeus Hunton et par W. E. B. Du Bois, et incarnera la voix majeure de l'anticolonialisme en Amérique (vON ESCHEN 1997 : 17-21). Elle fut dissoute en 1955. La National Association for the Advancement of Colored People fut créée en 1909 pour défendre les droits civiques des Noirs américains et dénoncer, dans de retentissants procès, les injustices qu'ils subissaient. Elle vient de fêter son centenaire.

34. Fédération de sociétés savantes fondée en 1919, pour financer des bourses de recherche post-doctorale dans les humanités et les sciences humaines.

35. Créé en 1916 pour répondre à un besoin de recherches appliquées, cet organisme gouvernemental rassemble l'Académie des Sciences, l'Académie des Sciences de l'Ingénierie et l'Institut de Médecine.

36. LOCKE (1950 : 175) écrivait encore, en 1950 : «Although for a long time now, I have urged African studies and comparative research, there still seems to be little lay or professional interest in it. Negro college centers, partly through lack of financial resources, but also, let us confess, by reason of unfortunate mental alienation and disinterest, have done very little ; and worse yet, Negro students have rarely gone in for anthropological study, either linguistic, anthropometric or cultural at other wellequipped and thriving centers, such as Yale, Chicago and Northwestern. " Sur les fondations et le développement des premiers programmes d'études africaines, dans le cadre des area studies, voir GERSHENHORN (2004 : 175-189).

37. Ce comité réunissait entre autres Jackson Davis, directeur-adjoint du General Education Board, Frederick Keppel, ancien président de la Carnegie Corporation, Thomas J. Jones, directeur de la branche éducative du Phelps Stokes Fund, Emory Ross (1887-1973), ancien missionnaire au Congo, et les Afro-américains Rayford Logan, Charles S.Johnson, Ralph Bunche et Channing Tobias (1882-1961), secrétaire du département du Travail pour les gens de couleur au sein de la Young Men Christian Association (JANKEN 1993 : 171).

38. "Les Membres des Nations Unies qui ont ou qui assument la responsabilité d'administrer des territoires dont les populations ne s'administrent pas encore complètement elles-mêmes reconnaissent le principe de la primauté des intérêts des habitants de ces territoires. Ils acceptent comme une mission sacrée l'obligation de favoriser dans toute la mesure possible leur prospérité, dans le cadre du système de paix et de sécurité internationales établi par la présente charte, et à cette fin (a) d'assurer, en respectant la culture des populations en question, leur progrès politique, économique et social, ainsi que le développement de leur instruction, de les traiter avec équité et de les protéger contre les abus; (b) de développer leur capacité de s'administrer elles-mêmes, de tenir compte des aspirations politiques des populations et de les aider dans le développement progressif de leurs libres institutions politiques [...]» (Charte des Nations Unies, chapitre XI, article 73). Dans le chapitre suivant, consacré au régime international de tutelle, l'article 76 redéfinit ainsi «les fins essentielles du régime de tutelle »: "favoriser le progrès politique, économique et 
social des populations des territoires sous tutelle ainsi que le développement de leur instruction; favoriser également leur évolution progressive vers la capacité à s'administrer eux-mêmes ou l'indépendance ». Dans une lettre privée à sa femme, en date du 17 juin 1945, Bunche écrit : "We have a Trusteeship Chapter in this Charter, tho many thought it could never be pulled off. It is not as good as I would like it to be, but better than any of expected it could get - there were long periods here when it seemed that we would lose and would have no Chapter at all. A good part of the phraseology, inc[luded] some of the most difficult provisions was drafted exclusively by me. It is a thrill even for your blasé old hubby to see his own writing in it » (cité dans URQUHART $1993: 121$ ).

\section{RÉSUMÉS}

Résumé

Cet article revisite les différents projets de voyage ou de recherche africanistes développés par Alain Locke, dans la première moitié $\mathrm{du} \mathrm{XX}$ siècle, et il s'interroge sur les raisons de leurs échecs successifs à l'Université d'Howard. Basée sur diverses archives, et sur des rééditions récentes, cette enquête historique vise à replacer le développement des African Studies dans leur contexte institutionnel et politique, tout en soulignant certains rapports de force et certaines filiations méconnues. Si les études africaines et afro-américaines sont, aux États-Unis, étroitement associées au nom de Melville Herskovits, ce dernier doit beaucoup à la démarche théorique et aux problématiques culturalistes initiées par Alain Locke et divers africanistes noirs américains. Mais en se focalisant, parallèlement, sur les diverses situations coloniales du monde noir, certains spécialistes afro-américains en sciences sociales ont également exercé une certaine influence dans la réforme des systèmes coloniaux : la carrière internationale de Ralph Bunche s'inscrit par exemple dans le prolongement des projets africanistes d'Alain Locke. En définitive, les relations entre Locke, Herskovits et Bunche révèlent quelques tensions inhérentes au projet afroaméricain d'études africaines, et constitutives de l'opposition entre africanisme et afrocentrisme.

Abstract

This paper presents the different travels and african studies projects proposed by Alain Locke during the first half of the twentieth century, and it explores the reasons behind their successive failures at Howard University. Based on archival work and recent editing of diverses manuscripts, this historical survey aims at replacing the development of African Studies in their institutional and political context, while underscoring unequal terms and underrated relations between black and white scholars. If African and African-American Studies are closely related to Melville Herskovits' name in the United States, the american anthropologist borrowed much from Alain Locke and other black Africanists. While focusing on different colonial situations of the black world, some african-american social scientists also influenced the reforming of colonial systems: the international career of Ralph Bunche can thus be seen for example as a concrete extension of Alain Locke's africanist projects. In the end, the relationships between Locke, Herskovits and Bunche reveal some characteristics of the african-american idea and practice of african studies, as well as some tensions between africanism and afrocentrism. 
INDEX

Keywords : Ralph Bunche, Melville Herskovits, Alain Locke, Decolonization, African Studies, Foundations, History and Sociology of Knowledge, Panafricanism, International Relations

Mots-clés : Ralph Bunche, Melville Herskovits, Alain Locke, décolonisation, études africaines, fondations, histoire et sociologie des connaissances, panafricanisme, relations internationales

\section{AUTEUR}

\section{ANTHONY MANGEON}

Institut de recherches intersites Études culturelles, Université Paul Valéry, Montpellier. 